\title{
Pulsed Photonuclear Assessment (PPA) Technique: CY-05 Project Summary Report
}

J.L. Jones

B.D. Bennett

K.J. Haskell

J.T. Johnson

D.R. Norman

J.W. Sterbentz

R.W. Watson

S.M. Watson

W.Y. Yoon

J.M. Zabriskie

C.E. Moss

K.L. Folkman

A.W. Hunt

C.C. O'Neill

R.J. Spaulding

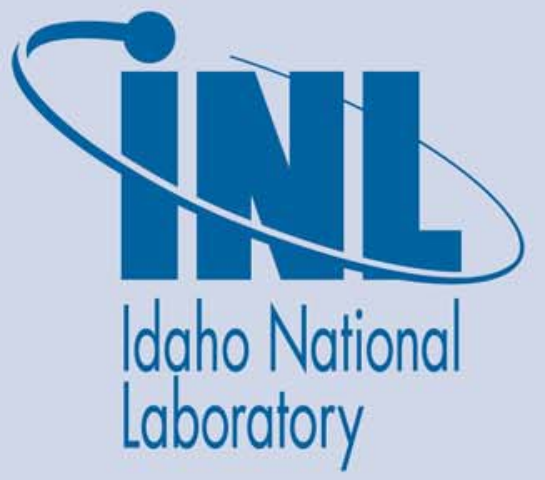

December 2005

The INL is a U.S. Department of Energy National Laboratory operated by Battelle Energy Alliance 

INL/EXT-05-01020

\title{
Pulsed Photonuclear Assessment (PPA) Technique: CY-05 Project Summary Report
}

\author{
J.L. Jones, B.D. Bennett, K.J. Haskell, J.T. Johnson, \\ D.R. Norman, J.W. Sterbentz, R.W. Watson, \\ S.M. Watson, W.Y. Yoon, J.M. Zabriskie \\ C.E. Moss, LANL \\ K.L. Folkman, A.W. Hunt, C.C. O'Neill, R.J. Spaulding, ISU \\ December 2005 \\ Idaho National Laboratory \\ National and Homeland Security \\ Idaho Falls, Idaho 83415 \\ Prepared for the \\ U.S. Department of Homeland Security by the U.S. Department of Energy \\ Under DOE Idaho Operations Office \\ Contract DE-AC07-05ID14517
}




\begin{abstract}
Idaho National Laboratory, along with Idaho State University's Idaho Accelerator Center and Los Alamos National Laboratory, is developing an electron accelerator-based, photonuclear inspection technology, called the Pulsed Photonuclear Assessment (PPA) system, for the detection of nuclear material concealed within air-, rail-, and, primarily, maritime-cargo transportation containers. This report summarizes the advances and progress of the system's development in 2005. The contents of this report include an overview of the prototype inspection system, selected Receiver-Operator-Characteristic curves for system detection performance characterization, a description of the approach used to integrate the three major detection components of the PPA inspection system, highlights of the gray-scale density mapping technique being used for significant shield material detection, and higher electron beam energy detection results to support an evaluation for an optimal interrogating beam energy. This project is supported by the Department of Homeland Security Office of Research and Development and, more recently, the Domestic Nuclear Detection Office.
\end{abstract}





\section{ACKNOWLEDGMENTS}

The authors want to express their thanks to Dr. Frank Harmon and Mr. Brett King of Idaho State University for their outstanding support of our project's accelerator development and testing needs at the Idaho Accelerator Center complex. 



\section{CONTENTS}

ABSTRACT

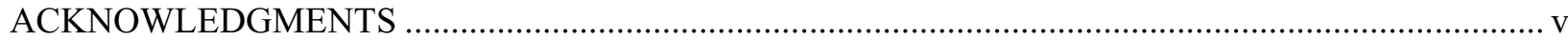

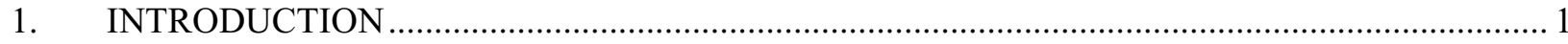

2. PROTOTYPE INSPECTION SYSTEM OVERVIEW …..................................................... 4

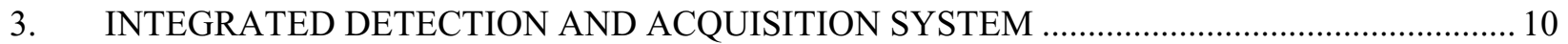

4. HIGHER ELECTRON BEAM ENERGY INTERROGATIONS ............................................... 16

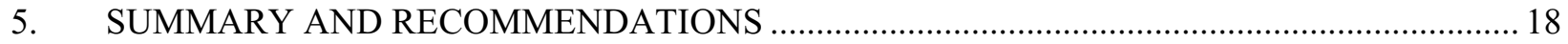

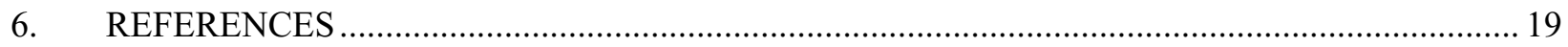

Appendix A-One-Step Versus Multi-Step Transport Calculation for PPA Applications Using the MCNPX Code

Appendix B - Single- Versus Multiple-Pulse Effects for the Pulsed Photonuclear Assessment (PPA)

Technique

\section{FIGURES}

1. The DHS PPA nuclear material detection/inspection system................................................. 1

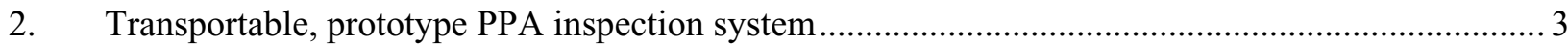

3. Cargo configuration schematic top view (left) and end view (right). Note the detector location numbers within "end view."

4. Photographs of the prototype PPA system with a cut away of a cargo container (left), and a metric ton of polyethylene being moved into position for a nuclear material inspection (right)

5. A reconfigurable test cell for indoor/outdoor operations with the PPA inspection system at the IAC/ITRDL complex. (The Varitron is inside the trailer and another similar accelerator is located in the smaller side test cell)

6. Neutron response ROC curves for selected PNDs (\#3, 4, and 16) with the Wood and Iron

Calibration Pallets

7. Gamma-ray response ROC curves for selected GM detectors (\#3, 4, and 16) with Wood and Iron Calibration Pallets 
8. Typical inspection time-integrated, GM detector gamma-ray responses (curves 1-7) and a bare He-3 detector neutron response (curve 8) after each accelerator pulse for a 120-s, 125$\mathrm{Hz}$ inspection without nuclear material

9. General layout diagram of the integrated nuclear material detection system (top) and the system's interfacing hardware (bottom)

10. PPA detection system user interface display.

11. Selected user interface display with a representative Calibration Pallet inspection (while the INL Celotex Calibration Pallet is pictured, the data presented is from a Lead Pallet inspection)

12. The user interface display showing the inspection data for the INL Polyethylene Calibration Pallet.

13. GSDM of the INL Calibration Pallets with and without the enclosing DU material

14. PACECO/ISU/INL gray scale density mapping system mounted with the IAC cargo container translation test assembly

15. X-ray "image" acquired with the gray scale density mapping system of a $12.2-\mathrm{m}(40-\mathrm{ft})$ cargo container

16. The energy-dependent PND response with and without the $4.8-\mathrm{kg}$ DU plates using various beam currents, accelerators, and testing locations/facilities. (Assumes a $\pm 10 \%$ systematic beam energy error and uses a perpendicular, 2-m DU-to-accelerator and DU-to-detector test configuration.)

17. The energy-dependent PND response for the INL Wood Calibration Pallet with and without the $4.8-\mathrm{kg}$ DU plates

B-1. Number of delayed neutrons counted in subsequent count intervals from a SINGLE accelerator pulse (Pulse No. 1)

B-2. Integrated delayed neutron counts from a SINGLE accelerator pulse (Pulse No. 1)

B-3. Actual MCS integration delayed neutron count curve (top curve), plus a hypothetical curve (lower curve) in which delayed neutron contributions from previous pulses are ignored. Note the large difference between the two curves indicative of the important role delayed neutrons from previous pulses play in the total count.....

\section{TABLES}

1. INL Calibration Pallet Data and Key Detection Information. 5

2. Cargo Container Inspection Delayed Neutron Count Data (Avg. of Detector Positions 3 and 16) for 120-s Inspections Using 4.8-kg DU and the INL Calibration Pallets 


\section{Pulsed Photonuclear Assessment Technique: CY05 Project Summary Report}

\section{INTRODUCTION}

In the 1970's, Idaho National Laboratory (INL) began evaluating an early application of a Pulsed Photoneutron Assessment (PPA) technique as a waste conveyor monitoring system. ${ }^{1}$ While Los Alamos National Laboratory (LANL) researchers have developed various neutron interrogation systems, such as a waste assay system ${ }^{2}$, they had also studied a PPA-type technique that used a $10-\mathrm{MeV}$ electron accelerator to obtain highly enriched uranium (HEU), natural uranium, and plutonium photofission-product spectra between accelerator pulses using a heavily-shielded, high-purity germanium detector. ${ }^{3}$ INL continued to apply the PPA technology to support treaty verification applications related to nuclear weapon configurations, which required the development of enhanced radiation detectors ${ }^{4-6}$ and the design and fabrication of a unique, transportable, energy-selectable electron accelerator- the INL Varitron. ${ }^{4}$ The PPA technique, in collaboration with LANL and the Idaho State University's Idaho Accelerator Center (IAC), has continued to support nuclear material detection in various challenging (shielded) configurations. $^{7-12}$ Currently, the Department of Homeland Security's (DHS) Science and Technology Directorate and the Domestic Nuclear Detection Office (DNDO) are fostering the development of an advanced nuclear material detection capability applicable to a wide variety of cargo-container types and loading configurations. ${ }^{13-17}$ While this report's predecessor ${ }^{14}$ identified and described the development of key detection components of the proposed DHS PPA inspection system, this CY05 follow-on report summarizes the integrated prototype system. Figure 1 is a schematic of the current prototype PPA inspection system for nuclear material detection.

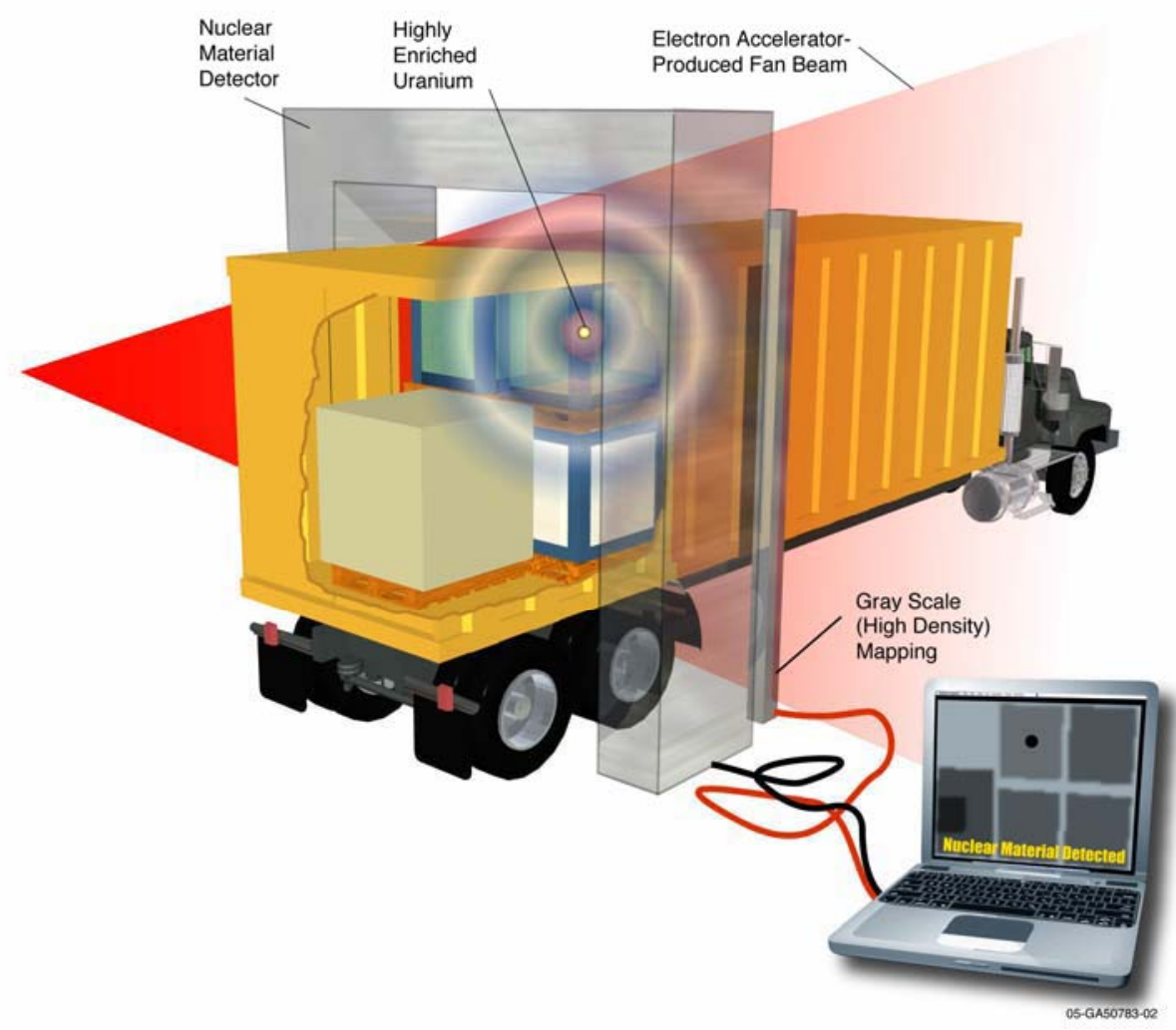

Figure 1. The DHS PPA nuclear material detection/inspection system. 
The major attributes of the DHS PPA inspection system include:

- High-sensitivity for detection with fission reactions in nuclear materials

- Complementary, high-resolution, commercially-available $\mathbf{x}$-ray radiography/imaging with photon interrogation

- $\quad$ Complementary external neutron interrogation capability using a photoneutron converter placed at the photon source

- Commercially-available electron accelerators used as the photon source

- High photon flux intensity from electron accelerators (typically four orders-of-magnitude more intense than neutron fluxes from commercial neutron generators)

- High-energy photon penetration through dense and/or thick cargo materials with significant photofission reaction yields in nuclear materials

- Complimentary photoneutron production within shield materials and subsequent neutroninduced fissions in concealed nuclear materials (in addition to photofission effects).

The most recent version of the delayed transportable, prototype nuclear material detection system is shown in Figure 2. It utilizes arrays of custom-built and commercially-available Photonuclear Neutron Detectors (PND) ${ }^{9}$ [patent-pending, 10-atm., helium-3 (He-3) tubes with unique electronics and shielding] and co-located, unshielded Geiger-Müller (GM) gamma-ray detectors [patent-pending, LND, Inc. tubes (Model 719) with electronics and acquisition software]. Also shown in Figure 2 is a representative inspection application utilizing a one-and-a-half metric-ton cargo (i.e., wood and polyethylene pallet loading) configuration positioned under the array of detectors. Inside the trailer, behind the detectors and the pallets, are the other major components of the prototype PPA system that includes the pulsed photon source and the data acquisition system. User controlled operation is provided from a remote location.

This report presents an overview of the PPA prototype inspection system, describes the integrated detection and acquisition system along with the newest, patent-pending, gray-scale density mapping (GSDM) system, provides additional higher electron beam energy detection responses and provides a summary with recommendations for continued research and development needs. 


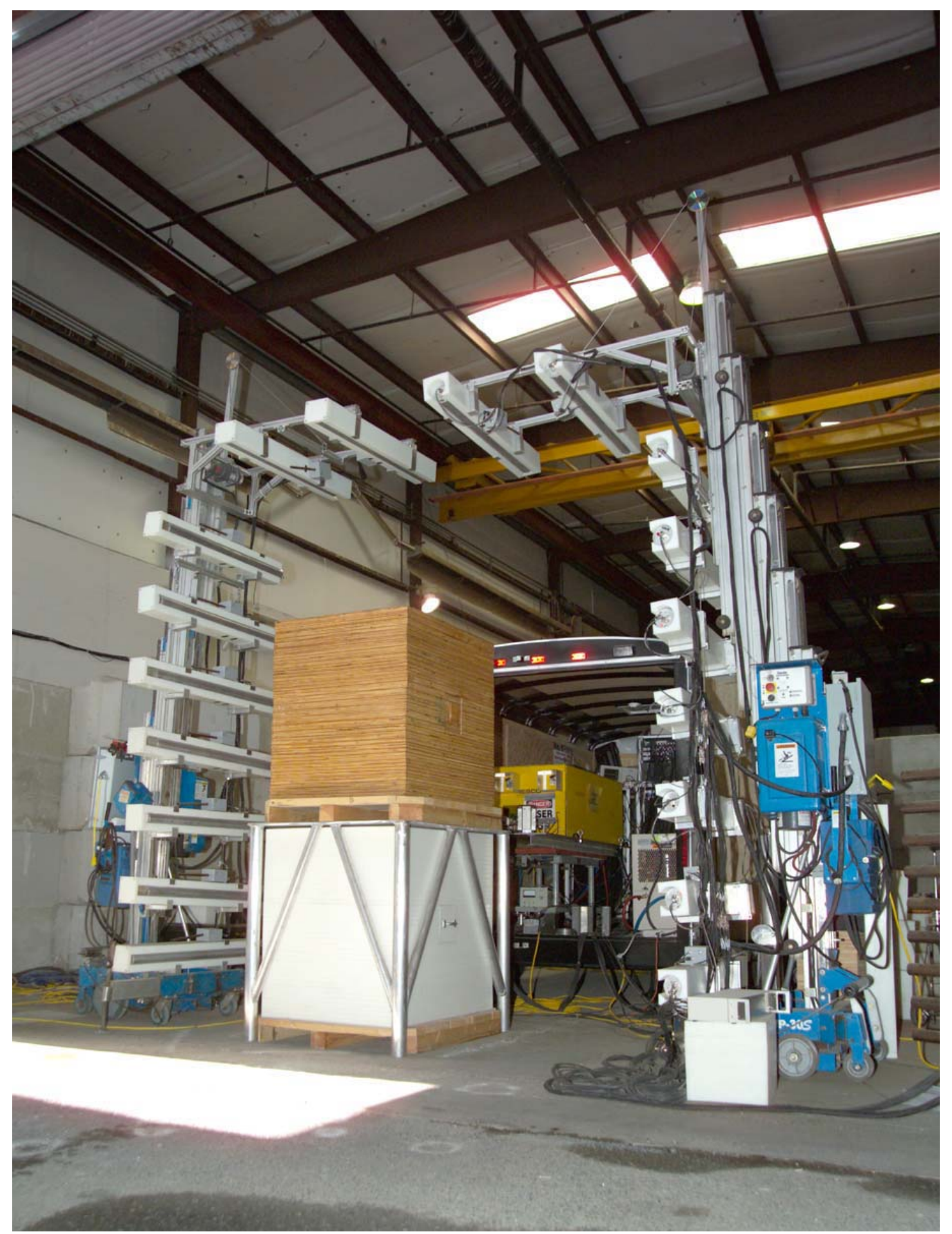

Figure 2. Transportable, prototype PPA inspection system. 


\section{PROTOTYPE INSPECTION SYSTEM OVERVIEW}

The developing prototype system has been shown to detect nuclear material in a series of seven INL Calibration Pallets ${ }^{14-16}$ even when these pallets are placed in a "challenging" location within a realistic cargo container configuration. ${ }^{17}$ The "challenging" inspection configuration is identified in Figure 3, along with the corresponding photographs in Figure 4. The nuclear material is placed in the center of the pallets and the pallet is positioned in the center of the cargo container. This inspection scenario represents a "challenge" because it provides both a realistic photon source-to-cargo center distance ( 2.5 to $3 \mathrm{~m}$ ) while maximizing the nuclear material-to-detector distances for all PND and GM detectors. For this cargo inspection configuration, the interrogating photon beam travels through the cargo at nearly a 45-degree angle and the radiation detectors are positioned along the sides of the cargo container.

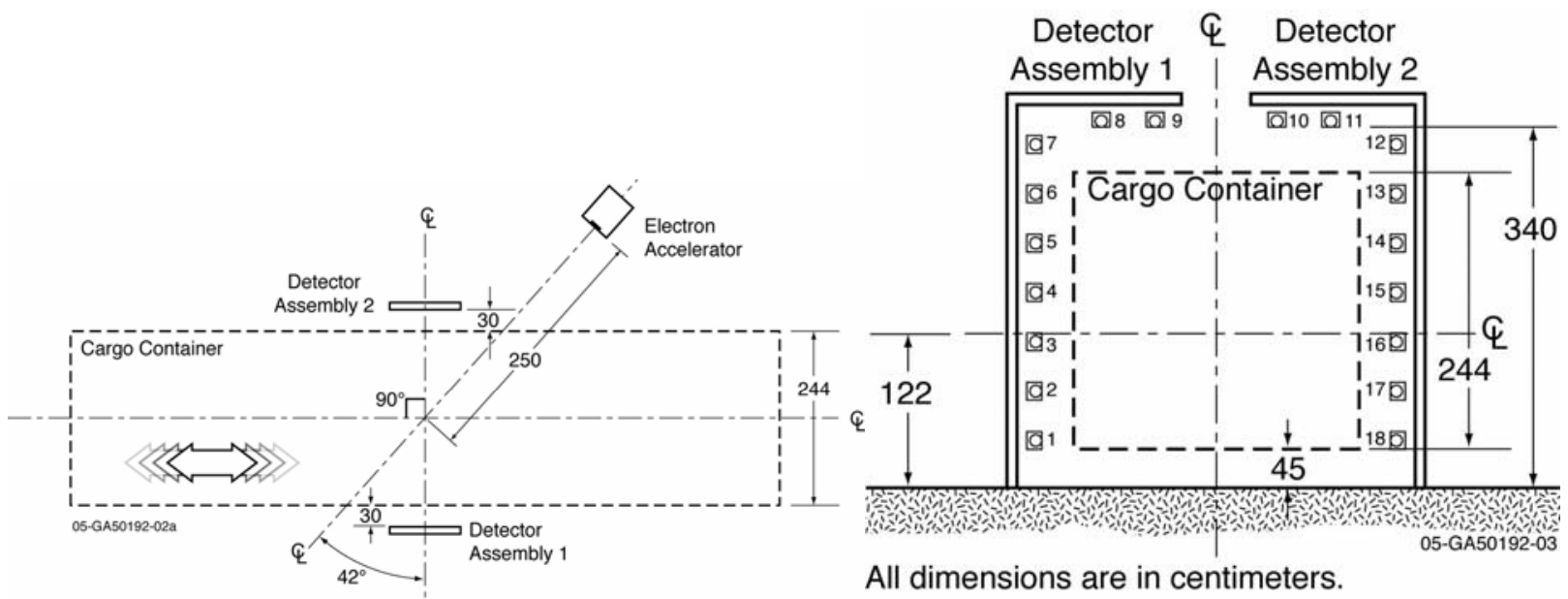

Figure 3. Cargo configuration schematic top view (left) and end view (right). Note the detector location numbers within "end view."
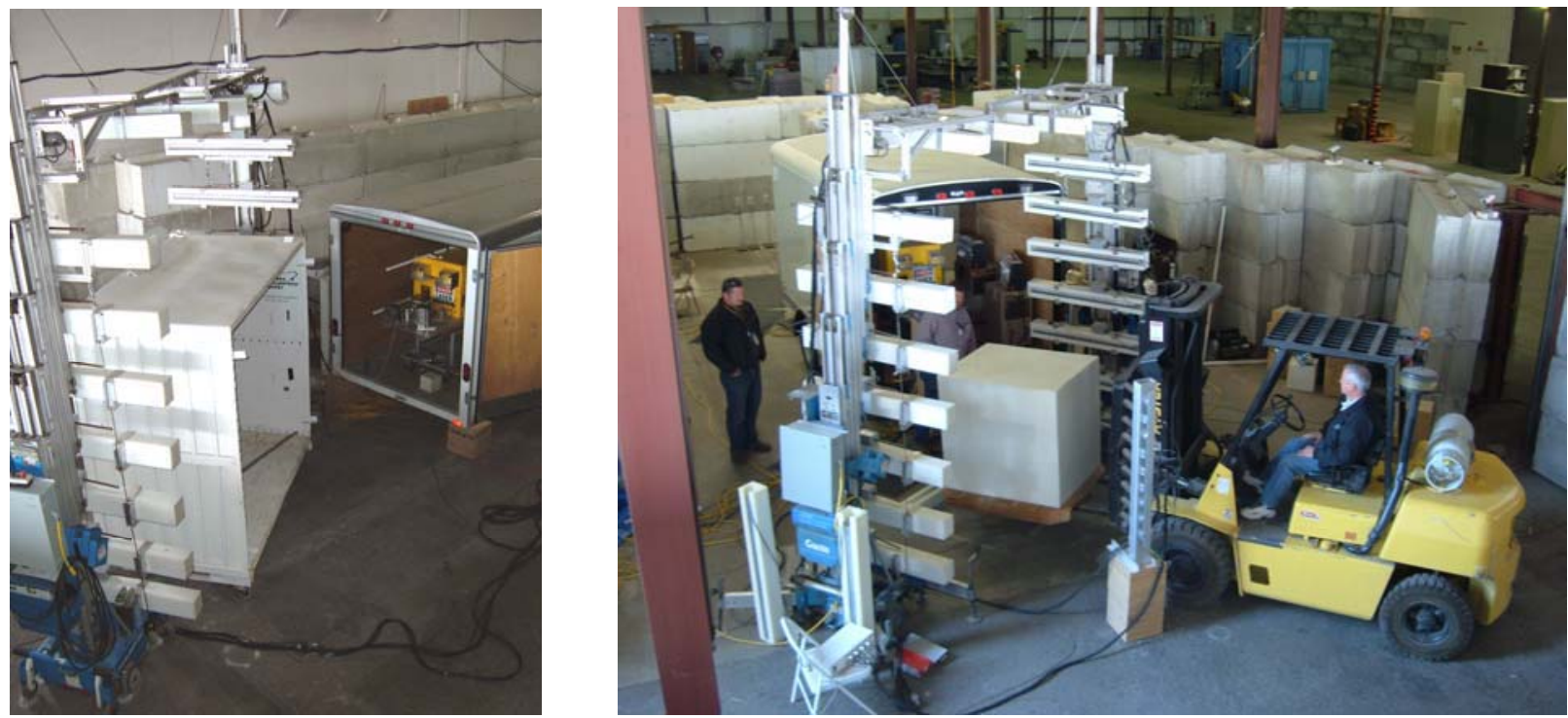

Figure 4. Photographs of the prototype PPA system with a cut away of a cargo container (left), and a metric ton of polyethylene being moved into position for a nuclear material inspection (right). 
For these inspection assessments, Table 1 presents pertinent information about the cargo type and the dominant detection effects. A 4.8-kg sample of depleted uranium (DU) was used as a representative nuclear material-of-interest. DU was selected since it is readily available, has a high probability of being encountered (potential false positive) during commercial cargo inspection applications, and will underestimate the system's performance for the detection of HEU. ${ }^{7}$ As shown in Table 1, DU was detected by neutron emissions in some cases, gamma-ray detection in others, and via both sets of detectors in three cases. It is important to note that the PPA system is designed such that any alarm (positive detection) response will provide a nuclear material detection event! Combining several similar detector responses has increased the nuclear material detection sensitivity in some cases.

Detailed description and experimental data are presented in Reference 17 regarding the realistic cargo container inspection configurations. Table 2 summarizes the overall delayed region-of-interest (ROI) detection data (acquired between 1.9 and $8 \mathrm{~ms}$ after each accelerator pulse) for $120-\mathrm{s}, 125-\mathrm{Hz}$ inspections and compares this experimental data to pre-test predictions. These predictions utilized the corresponding acquisition time and a similar configuration but with some important differences in accelerator standoff distance and angle, DU orientation, and detector locations. Hence, the predictions values are best-estimate values. However, even with these uncertainties, most of the neutron and gammaray predictions are well within a factor of two of the experimental results, and hence, final post-test evaluations were not deemed necessary. For the Empty and Celotex Pallet cases, the slightly larger differences are attributed to the positioning of the DU material within the Empty Pallet and the use of an incorrect effective material density with the Celotex Pallet, respectively.

All calculated pallet responses are based on Monte Carlo calculations using a multi-step INL methodology ${ }^{14}$ that incorporates the MCNPX radiation transport code. ${ }^{18}$ Appendixes A and B, respectively, present information regarding the INL calculation methodology and a clarification between single- versus multiple-pulse PPA inspection effects in resolving differences in experimental and predictive results.

Table 1. INL Calibration Pallet Data and Key Detection Information.

\begin{tabular}{|c|c|c|c|c|c|c|c|}
\hline Pallets & $\begin{array}{c}\text { Mass } \\
\text { (metric ton) }\end{array}$ & $\begin{array}{l}\text { Primary } \\
\text { Material }\end{array}$ & $\begin{array}{c}\text { Material } \\
\text { Density } \\
(\mathrm{g} / \mathrm{cc})\end{array}$ & $\begin{array}{c}\text { Pallet } \\
\text { Dimensions } \\
(\mathrm{cm})\end{array}$ & $\begin{array}{l}\text { Primary } \\
\text { Detection } \\
\text { Challenge } \\
\end{array}$ & $\begin{array}{c}\text { Detection } \\
\text { with } \\
\text { Neutrons }\end{array}$ & $\begin{array}{c}\text { Detection } \\
\text { with } \\
\text { Gammas } \\
\end{array}$ \\
\hline $\begin{array}{l}\text { Empty } \\
\text { (cylindrical } \\
\text { annulus) } \\
\end{array}$ & 0.0 & Aluminum & 2.70 & 0.6 (thick) $\times 20$ & None & Yes & Yes \\
\hline Celotex & 0.05 & "Celotex TM" & 0.05 & $107 \times 87 \times 102$ & None & Yes & Yes \\
\hline $\begin{array}{l}\text { Borated } \\
\text { Polyethylene }\end{array}$ & 1.0 & $\begin{array}{l}5 \% \text {-Borated } \\
\text { polyethylene }\end{array}$ & 1.05 & $107 \times 87 \times 102$ & $\begin{array}{l}\text { Neutron } \\
\text { Moderation } \\
\text { and } \\
\text { Absorption }\end{array}$ & No & Yes \\
\hline Polyethylene & 0.9 & Polyethylene & 0.95 & $107 \times 87 \times 102$ & $\begin{array}{l}\text { Neutron } \\
\text { Moderation }\end{array}$ & No & Yes \\
\hline Wood & 0.5 & Plywood & 0.48 & $107 \times 87 \times 102$ & $\begin{array}{l}\text { Neutron } \\
\text { Moderation }\end{array}$ & Yes & Yes \\
\hline $\begin{array}{l}\text { Lead } \\
\text { (cylindrical } \\
\text { annulus) } \\
\end{array}$ & 0.2 & Lead & 10.80 & 5 (thick) by 28 & $\begin{array}{l}\text { Photon } \\
\text { Attenuation }\end{array}$ & Yes & No \\
\hline $\begin{array}{l}\text { Iron } \\
\text { (cylindrical } \\
\text { annulus) }\end{array}$ & 0.9 & Iron & 7.80 & $\begin{array}{l}16 \text { (thick) by } \\
61\end{array}$ & $\begin{array}{l}\text { Photon } \\
\text { Attenuation }\end{array}$ & Yes & No \\
\hline
\end{tabular}


Table 2. Cargo Container Inspection Delayed Neutron Count Data (Avg. of Detector Positions 3 and 16) for 120-s Inspections Using 4.8-kg DU and the INL Calibration Pallets.

\begin{tabular}{|l|c|c|c|c|}
\hline \multirow{2}{*}{ Pallet Type } & \multicolumn{2}{|c|}{ Net Neutron Counts } & \multicolumn{2}{c|}{ Total Gamma-ray Counts } \\
\cline { 2 - 5 } & Experimental & $\begin{array}{c}\text { Ratio } \\
\text { (Calculated/ } \\
\text { Experiment) }\end{array}$ & $\begin{array}{c}\text { Ratio } \\
\text { (Calculated/ } \\
\text { Experiment) }\end{array}$ \\
\hline Empty & 2589 & 0.29 & 776 & - \\
\hline Celotex & 2421 & 0.28 & 472 & 0.91 \\
\hline Wood & 107 & 0.92 & 326 & 1.0 \\
\hline Lead & 145 & 0.57 & - & - \\
\hline Iron & 46 & 0.65 & - & 0.7 \\
\hline Poly. & - & - & 148 & 1.2 \\
\hline Borated-Poly. & - & - & 99 & - \\
\hline
\end{tabular}

To support field deployment of these PPA inspection systems, a reconfigurable open test cell configuration (shown in Figure 5) has been established at the IAC's Inspection Technology Research and Development Laboratory (ITRDL). This open test cell, with a 5-m wide door access, is ideal for supporting both large and small cargo inspection applications and supports both indoor and outdoor tests. To facilitate the transportability of the PPA inspection technology, the accelerator used to generate the interrogating photons is mounted in an enclosed, $\sim 5-\mathrm{m}$ long trailer. The enclosed trailer not only provides system transportability, but also shelters the system from inclement weather.

Also shown in Figure 5 is a side test cell. This cell includes a Varitron-like electron accelerator (the Varitron II) allowing other types of experimental PPA-related support. The Varitron II, built by the IAC, has a $2.5 \mathrm{E}-3 \mathrm{~cm}$ thick, stainless steel vacuum window (i.e., electron window) and is optionally equipped with changeable tungsten electron/photon converters, having selectable thicknesses ranging from 2.54E-2 to $2.79 \mathrm{E}-1 \mathrm{~cm}$. Also, the Varitron II has a more "radiation safe" design than the Varitron accelerator, has a coaxial solenoid design for enhanced electron beam optics, and incorporates a novel, low-volume, prototype energy analyzer for beam analyses.

For the Wood and Iron Calibration Pallet inspections, Receiver Operating Characteristic (ROC) curves (generated using the Analyse-it ${ }^{\circ}$ Software, Version 1.71, developed by Analyse-It Software, Ltd. Leeds, England) are shown in Figure 6 for three separate PND locations. As can be seen, the ROC curves for any one of these detector locations show a perfect nuclear material detection response (i.e., 100\% detection with $0 \%$ false positives). For comparison, the delayed gamma response ROC curves are shown in Figure 7. These curves are measured with GM detectors that were co-located with the PNDs described in Figure 6. The gamma-ray response also shows perfect ROC curves for the Wood Pallet; however, as expected, the Iron Pallet curves show that detector locations 4 and 16 provide some detection capability while detector location 3 has no discrimination/detection ability. Overall detection performances can be enhanced by combining GM detectors responses. The applicability and implementation of this enhancement is being assessed.

While GM detectors have increased the system's detection capability (especially for low density, hydrogenous cargo types), there are some complexities related to gamma-ray counting in pulsed photonuclear environments. One complexity is the copious neutron production associated with each interrogating photon pulse that induces time- and material-dependent gamma-ray emissions from an inspected cargo. The total delayed gamma-ray environment will depend on the amount and type of cargo. 


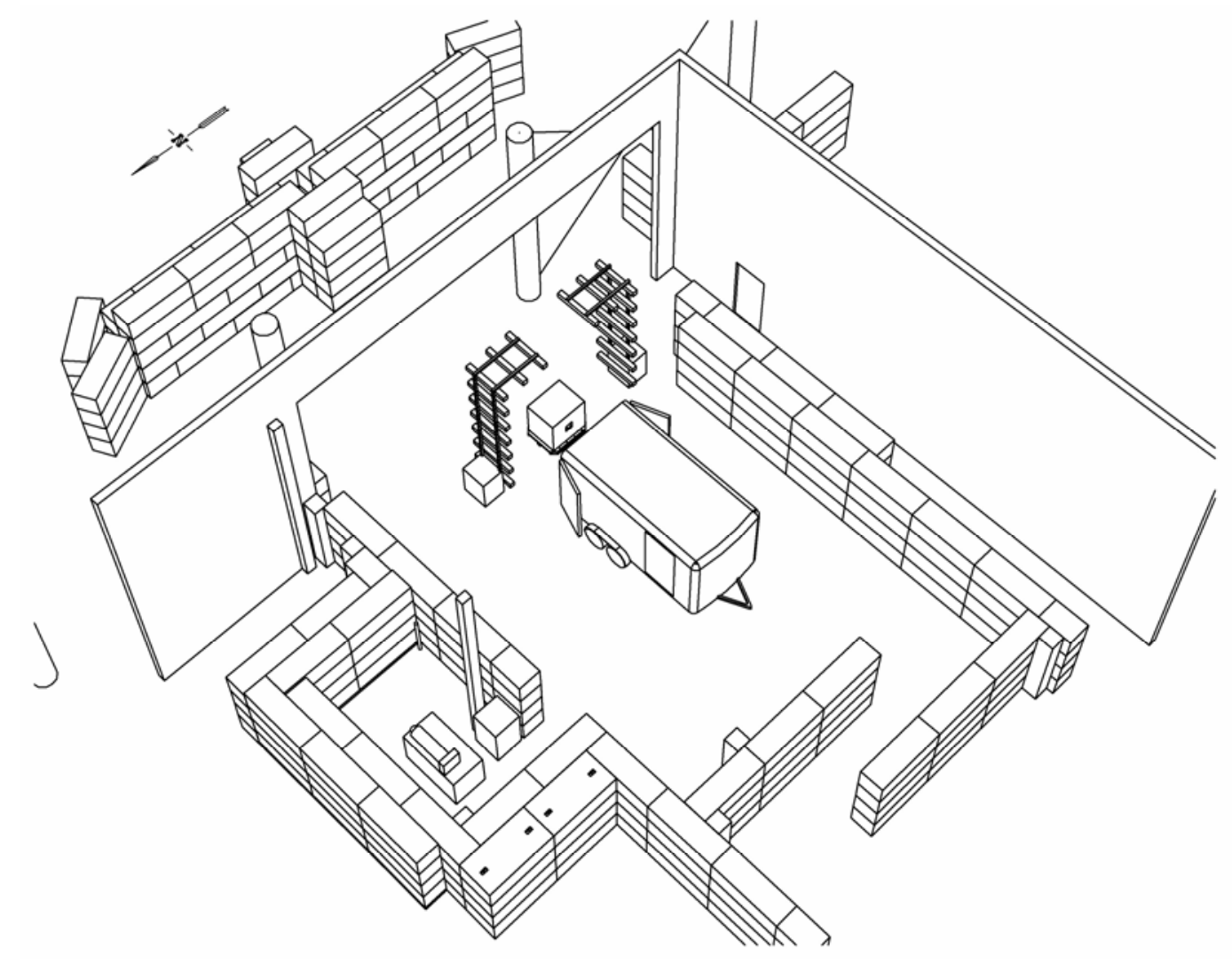

Figure 5. A reconfigurable test cell for indoor/outdoor operations with the PPA inspection system at the IAC/ITRDL complex. (The Varitron is inside the trailer and another similar accelerator is located in the smaller side test cell.)
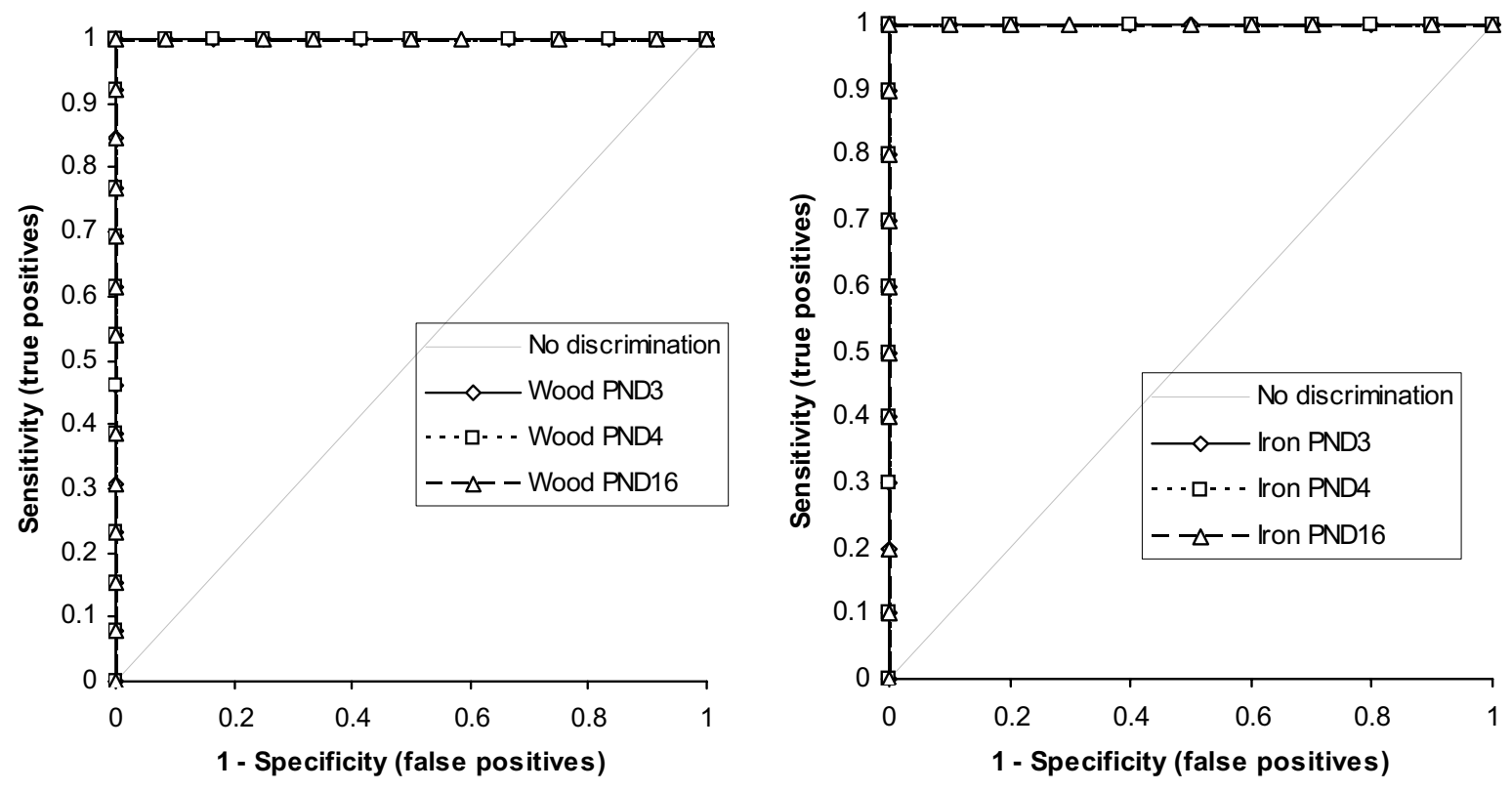

Figure 6. Neutron response ROC curves for selected PNDs (\#3, 4, and 16) with the Wood and Iron Calibration Pallets. 

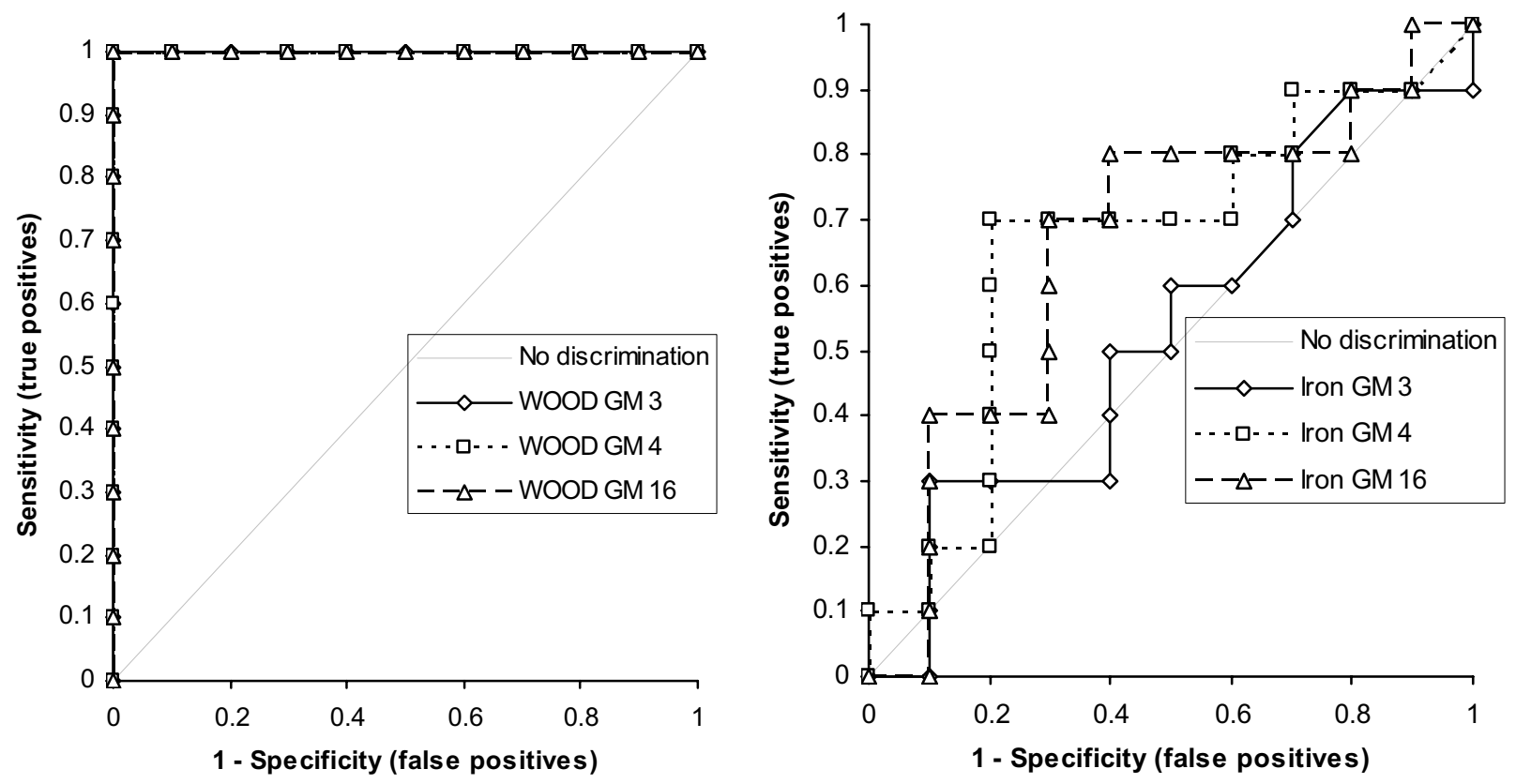

Figure 7. Gamma-ray response ROC curves for selected GM detectors (\#3, 4, and 16) with Wood and Iron Calibration Pallets.

However, a few milliseconds after each interrogating pulse, the resulting thermal neutron environment dominates the background gamma emission and results in an active gamma-ray background that could easily mask the induced gamma emissions from a concealed nuclear material. An improved signature method is proposed that attempts to compensate for this actively induced, gamma-ray background. This method utilizes the response of a bare He-3 detector, co-located with the PPA detector arrays, to monitor the thermal neutron (see Figure 8, plot number 8) environment $\left(\mathrm{n}_{\mathrm{th}}\right.$ ), and integrates the time-dependent gamma-ray response (see Figure 8, plots 1 thru 7) for each GM detector $(\gamma$ ). This signature method (see Equation 1) develops a time ( $\mathrm{t}$ )-dependent Figure-of-Merit (FOM) that is mathematically evaluated after each accelerator pulse $\left(t_{p}=\right.$ time after each pulse corresponding to a selected delayed ROI) and compares it to a predetermined, detection-based, time-dependent signature.

$$
\operatorname{FOM}(t)=\int_{0}^{t} \frac{\int_{t_{p}}^{1 / H z}\left(t_{p}\right) d t_{p}}{\int_{t_{p}}^{1 / H z} t_{t_{p}}\left(t_{p}\right) d t_{p}} d t
$$

This method relies on the fact that gross, time-dependent, thermal neutron capture response, measured by a GM tube per induced thermal neutrons during a pulse inspection, is relatively insensitive to changing cargo loadings, especially after several milliseconds after each accelerator pulse. Hence, any additional delayed gamma-ray contribution from a nuclear material should increase the value of the FOM. 
Other methods (e.g., optimization of the delayed gamma "region of interest") and hardware can be used to accomplish a similar detection objective and continue to be investigated.

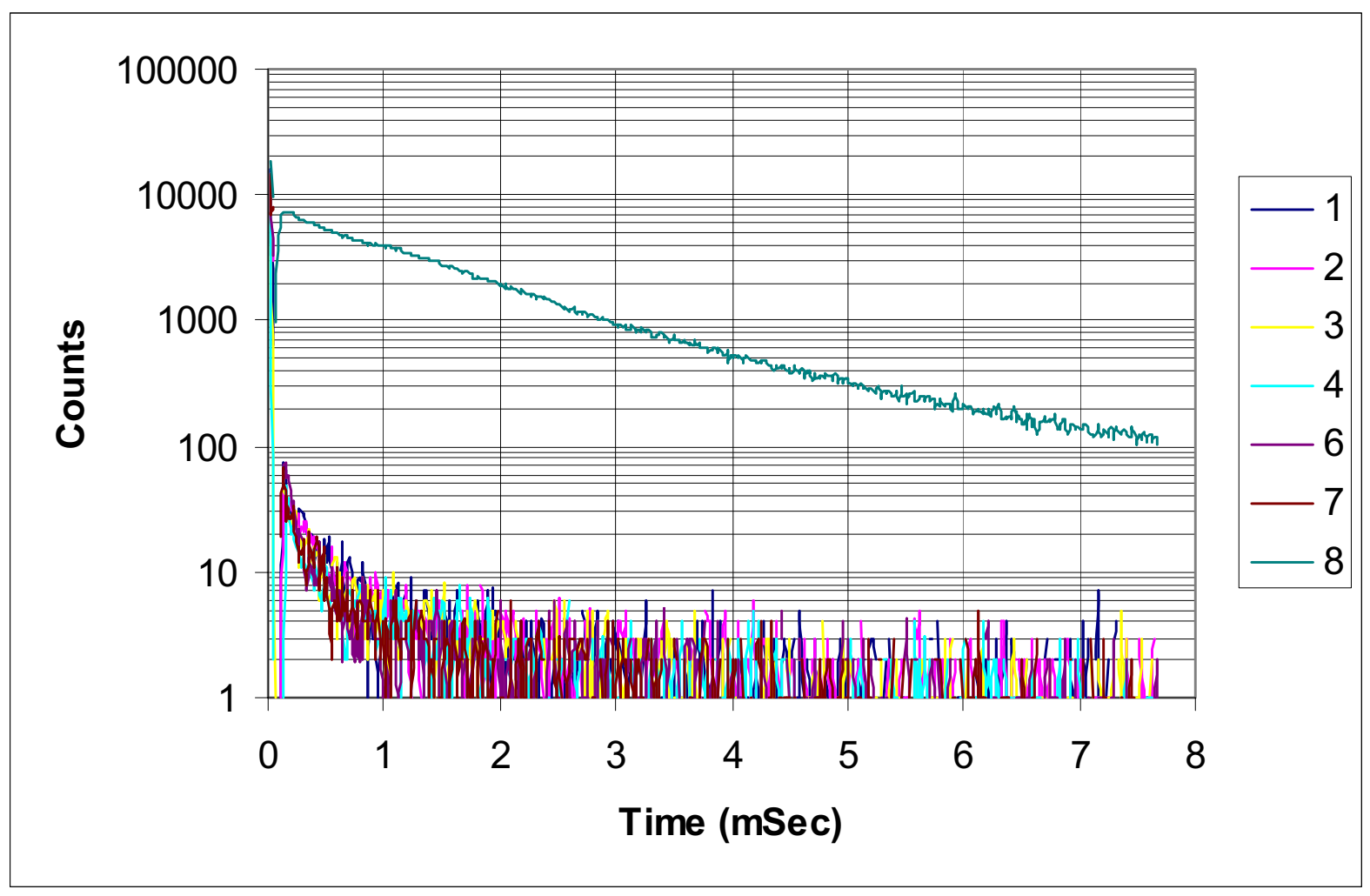

Figure 8. Typical inspection time-integrated, GM detector gamma-ray responses (curves 1-7) and a bare He-3 detector neutron response (curve 8) after each accelerator pulse for a $120-\mathrm{s}, 125-\mathrm{Hz}$ inspection without nuclear material.

It must be emphasized that GM detectors were initially selected as an inexpensive gamma-ray detection method to complement the optimized neutron-based detection system. While they, along with novel detection algorithms, are proving themselves to be operationally applicable within this type of pulsed photonuclear inspection environment, delayed gamma-ray detection within the PPA environment still needs to optimized. It is proposed that an assessment be undertaken showing the improvements from combined GM detector responses (including faster responding tubes) and the comparable benefits from enhanced gamma-ray detection technologies, such as fast scintillators, PIN diodes, higher resolution detectors, etc. 


\section{INTEGRATED DETECTION AND ACQUISITION SYSTEM}

An integrated detection and acquisition system has been developed for the DHS PPA inspection system that provides automated nuclear material detection capabilities. This integrated detection and acquisition system is incorporated in a very flexible National Instruments ${ }^{\mathrm{TM}}$ platform, using multiple Field Programmable Gate Array (FPGA) Modules and analog-to-digital converters, to support the operation of various types of radiation detectors. The nuclear material detection system integrates neutron, gamma-ray, and density mapping information into a single user-controlled interface. Figure 9 presents a diagram of the general layout of the integrated system and shows a photo of the interfacing hardware (located with the interrogating accelerator).

The integrated system acquires data from at least 18 PNDs monitoring the neutron emissions from an interrogated cargo loading and compares it to real-time, actively induced, neutron background signal to determine if nuclear material is present. The actively induced background neutrons are monitored with four PNDs, located near the primary PND arrays, but having their polyethylene shroud openings facing away from the cargo loading emissions. In addition, there are $18 \mathrm{GM}$ detectors, co-located with the PNDs, for monitoring the gamma-ray emissions from the nuclear materials. Finally, for the prototype system, there are $9 \mathrm{GM}$ detectors operating in the proportional region that are used for a patent-pending, GSDM method. These transmission mapping detectors are used to detect high-density (or a significant amount of low-density) shielding materials in cargo loadings. The ionizing-type radiation detection tubes have been selected due to their operational simplicity and their inherent interaction and recovery processes. The final two inputs are from a bare He-3 tube (also co-located with the system's radiation detectors) to monitor thermal neutrons and a trigger pulse from the Varitron accelerator to initialize the time-dependent counting response.

Figure 10 shows a representative user interface display for the PPA detection system. The display integrates the various radiation detection technologies. The center of the display is a schematic of a static cargo container. As nuclear material is detected by any GM or PND radiation detector, a flashing red box is overlaid onto the container image showing the time of detection (e.g., 40 seconds in the example shown). Surrounding this cargo container schematic, are two layers of detector counting boxes. The inner layer corresponds to the GM detectors whereas the outer layer corresponds to the PNDs. Also, a set of detectors can provide detection from under the cargo container in some cases, if applicable. The monitoring of the real-time, actively induced neutron background data is shown in the lower right of Figure 10. This data is used to define the system's detection limits. A time-dependent GSDM display of the actual cargo loading is shown directly below the count data. The second component of the GSDM system provides a shield material detection alarm. This alarm component is the multi-segmented bar, shown to the right of the count data, which corresponds to each of the transmission mapping detectors. A detector alarm is highlighted (red) when significant shield material is present (relative to a user-preset threshold value) during any portion of an inspection and indicates further inspection is mandated.

To further assist in understanding the user display response, Figure 11 focuses on only a portion of the display and includes a picture of an inspected INL Calibration Pallet. The photo has been sized to show the co-located PND and GM detector alignments with the surrounding detector boxes. The count data correspond to the detector locations identified in Figure 3. Note, Figure 11 also shows the PPA transmission mapping detector array to the right of the INL Calibration Pallet.

For the PND detectors, an alarm is triggered when they measure a signal that is significantly larger than the average response being measured by the background monitoring detectors. An alarmed state is shown by a highlight (red) around the counting box corresponding to that detector. Another alarm condition considers the summed response of groups of three consecutive PND detectors to provide increased sensitivity for diffuse/well-shielded sources. When this summed response alarms, a bar next to 


\section{Integrated System Hardware Diagram}

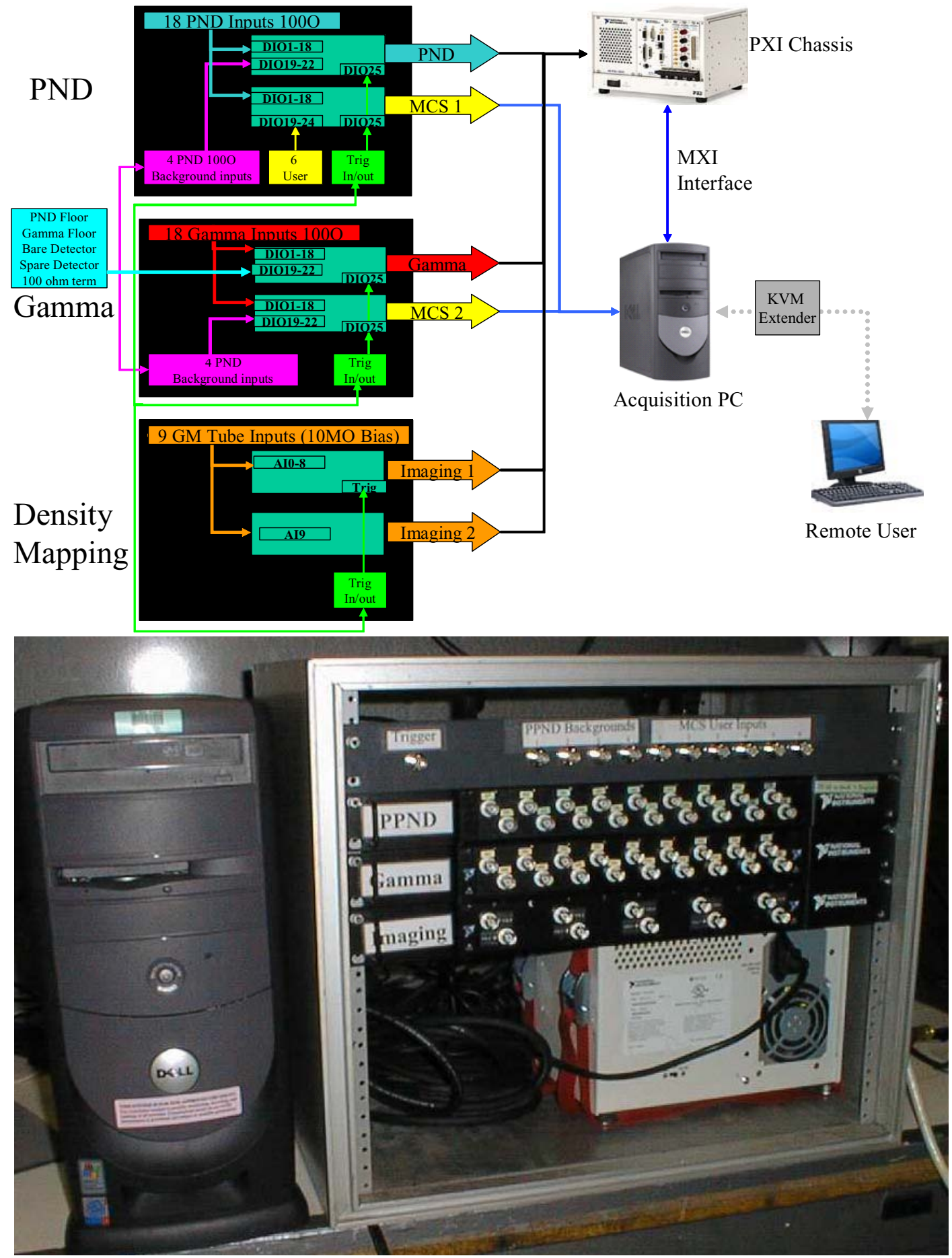

Figure 9. General layout diagram of the integrated nuclear material detection system (top) and the system's interfacing hardware (bottom). 


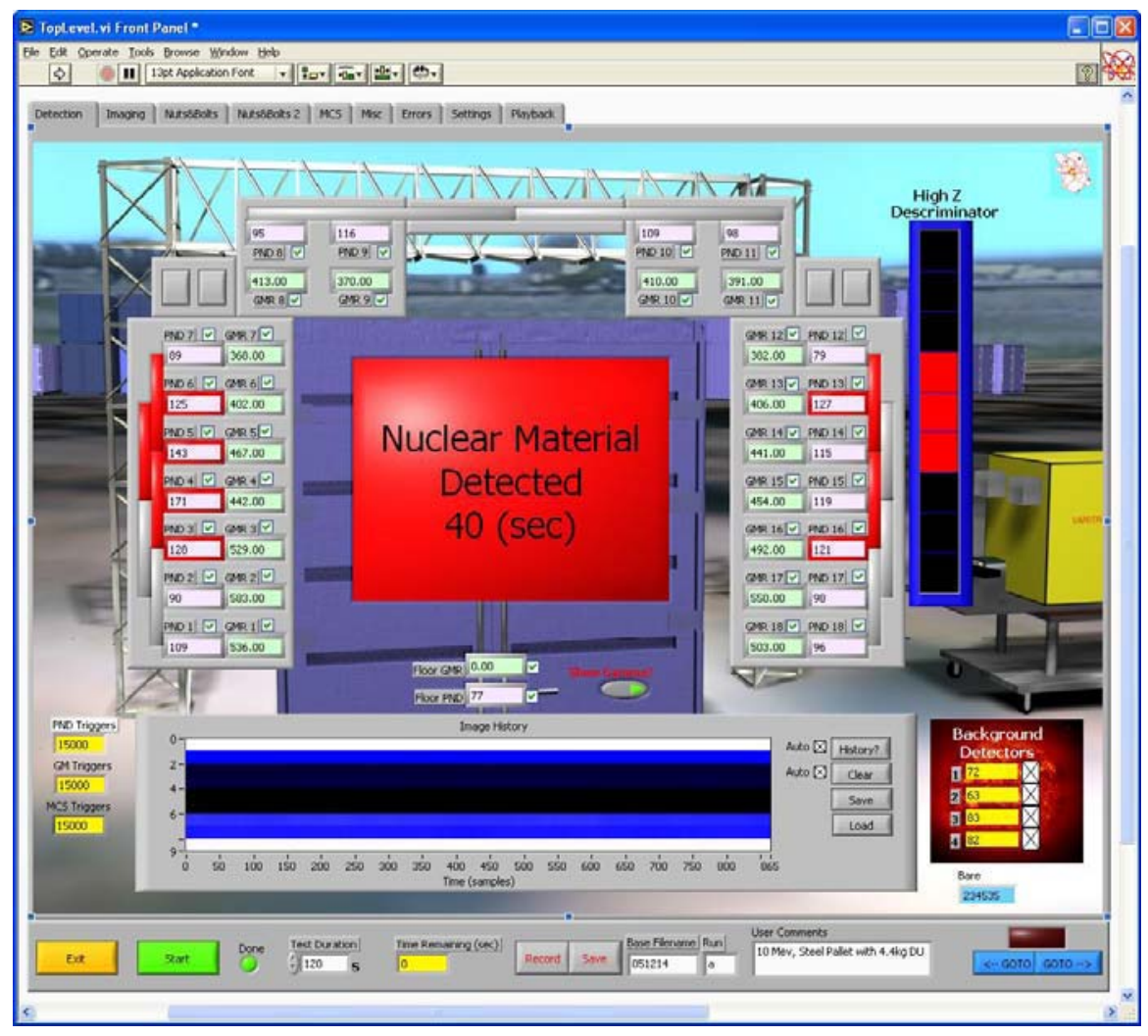

Figure 10. PPA detection system user interface display.

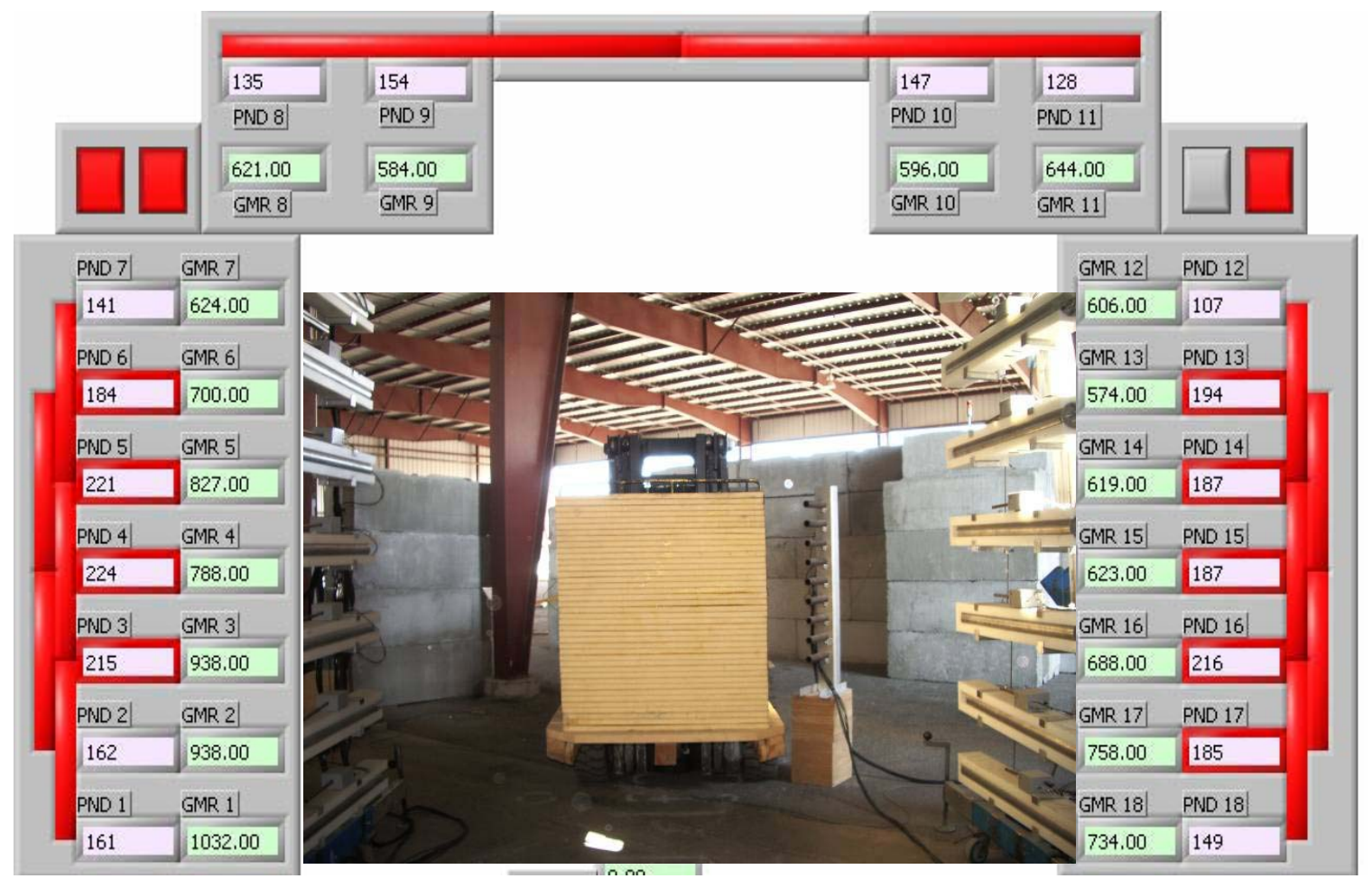

Figure 11. Selected user interface display with a representative Calibration Pallet inspection (while the INL Celotex Calibration Pallet is pictured, the data presented is from a Lead Pallet inspection). 
the group of PND detectors is highlighted (red). Note, when transitioning from the top- to the sidedetector locations, the summed group indicator changes from a bar to a pair of small boxes located at the upper corners of the display. For the $120-\mathrm{s}, 125-\mathrm{Hz}$ inspection data of the Lead Calibration Pallet (with DU) presented in Figure 11, only 9 out of 18 PNDs have individually alarmed, yet all group detectors have alarmed except for the upper right corner group (detectors 10 thru 12). Figure 10 shows similar display response data for a $120-\mathrm{s}, 125-\mathrm{Hz}$ inspection of the Iron Calibration Pallet containing the 4.8-kg DU sample. As can be seen by the fewer detector alarm responses in Figure 10, the Iron Pallet presents a more challenging 120-s inspection configuration than the Lead Pallet.

For the GM detectors, an alarmed state will correspond to a highlighted (red) border around a detector's counting box as shown in Figure 12. This figure shows a representative display for an inspection of the INL Polyethylene Calibration Pallet. The GSDM time-dependent display (below the counting data) shows the differences observed when DU is inserted in the polyethylene halfway thru the sampling process. (Note the 120-s counting data shown is acquired after the DU insertion.) Also, as seen by the multi-segment alarm bar, the shield material detector alarm has been activated. The current PPA system uses a simple count threshold for each GM detector. Based on the GM detection methodology discussed, and other options presented in the previous section, further increases in the detection sensitivity should be pursued.

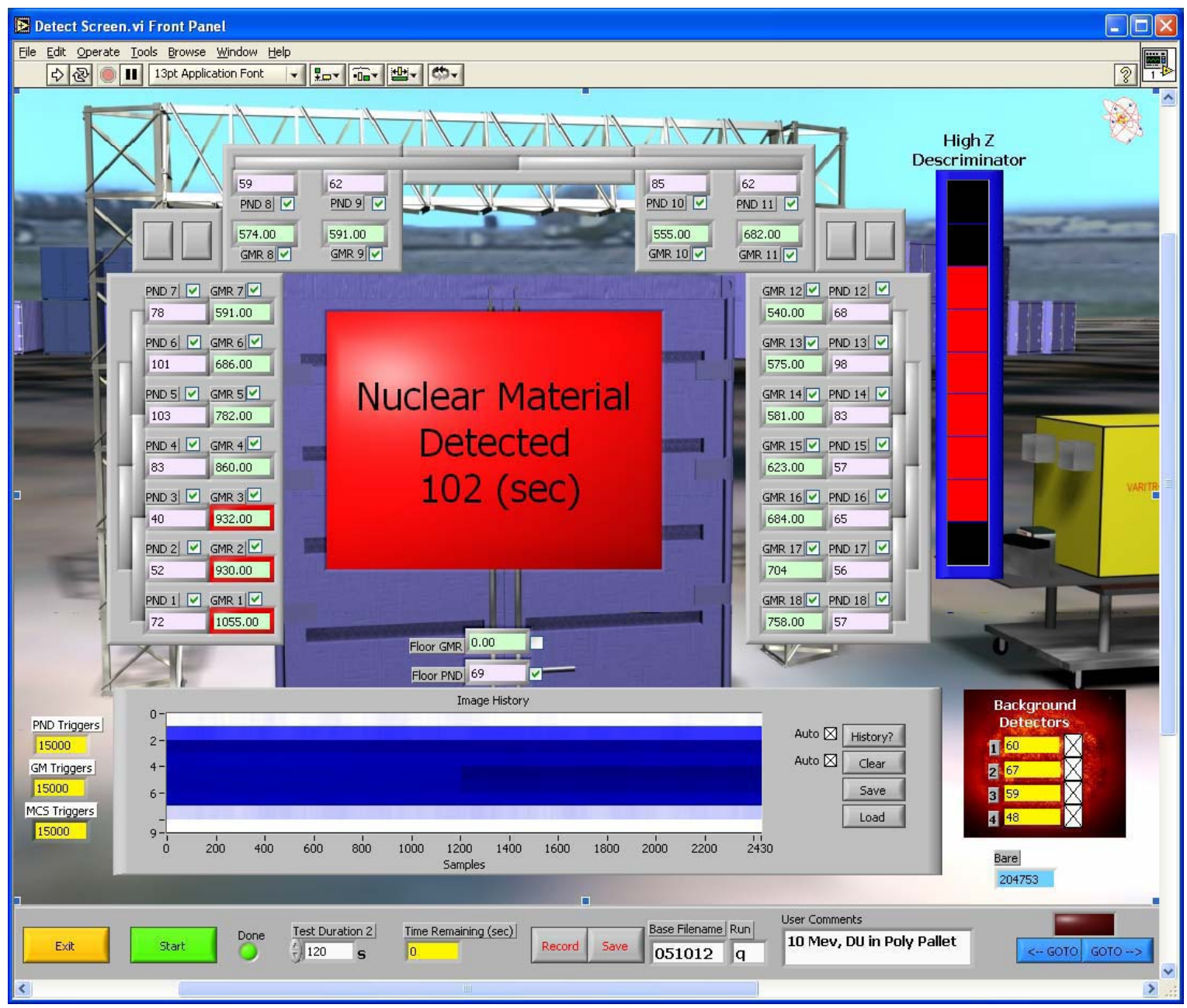

Figure 12. The user interface display showing the inspection data for the INL Polyethylene Calibration Pallet. 
As indicated previously, immediately beneath the integrated counting data on the user display is the time-dependent GSDM information corresponding to the cargo loading located between the interrogating photon source and the transmission mapping detector array. Figure 13 shows the typical GSDM results corresponding to most of the Calibration Pallets (with and without DU present) when positioned in the typical PPA-applicable cargo container inspection configuration. As can be seen from left to right, the effective pallet (or shield) densities are increasing. The dynamic range of the GSDM system (as deployed) is shown by the ability to discriminate the nuclear material within the cargo. The Polyethylene Pallet density begins to show signs of significant shielding and discrimination appears to be prohibited in the Lead and Iron Pallets. As shown in the Figures 10 and 12 the GSDM display (labeled "High Z Discrimination"), the alarm threshold was exceeded for the Iron and Polyethylene Calibration Pallet respectively. Clearly one can readily recognize the difficulty of using this simple mapping approach for imaging; however, the purpose of this mapping system is not to image nuclear materials, but rather detect the presence of significant shielding!

The Idaho Accelerator System, in partnership with INL, developed another version of this patentpending GSDM system for PACECO/Casper-Phillips. This system, consisting of a 16-detector, linear array of ionization tubes each separated by about $5 \mathrm{~cm}$ (see Figure 14), was initially tested at the IAC with a $12.2-\mathrm{m}$ (40-ft) cargo container moving at speeds up to $0.2 \mathrm{~m} / \mathrm{s}(0.65 \mathrm{ft} / \mathrm{s})$. While still focusing on shield material detection, the sensitivity of the overall GSDM system was assessed. Figure 15 presents the aerial density "image" obtained for this case. Since the bottom of the detector array was positioned about $30 \mathrm{~cm}$ from the floor of the cargo container, Figure 15 shows the resulting gray scale mapping of the upper portions of the Polyethylene and Lead Calibration Pallets. It is interesting to note that Figure 15 also identified a plastic chair!

These transmission "images" are only resolution limited by the tube's dynamic range and the tubeto-tube spacing. These tubes are very inexpensive and their overall detection inefficiencies are more than balanced by the specific intensity of each interrogating photon pulse. As shown previously, establishing a response threshold in each detector's output response appears to enable the detection of dense objects and/or the presence of significant shielding.

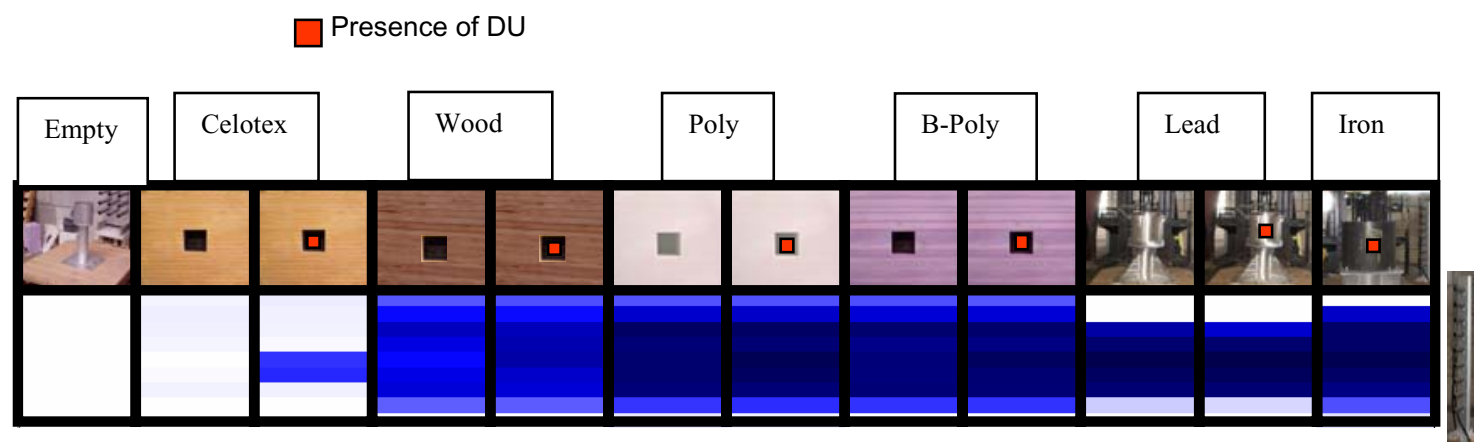

Figure 13. GSDM of the INL Calibration Pallets with and without the enclosing DU material. 


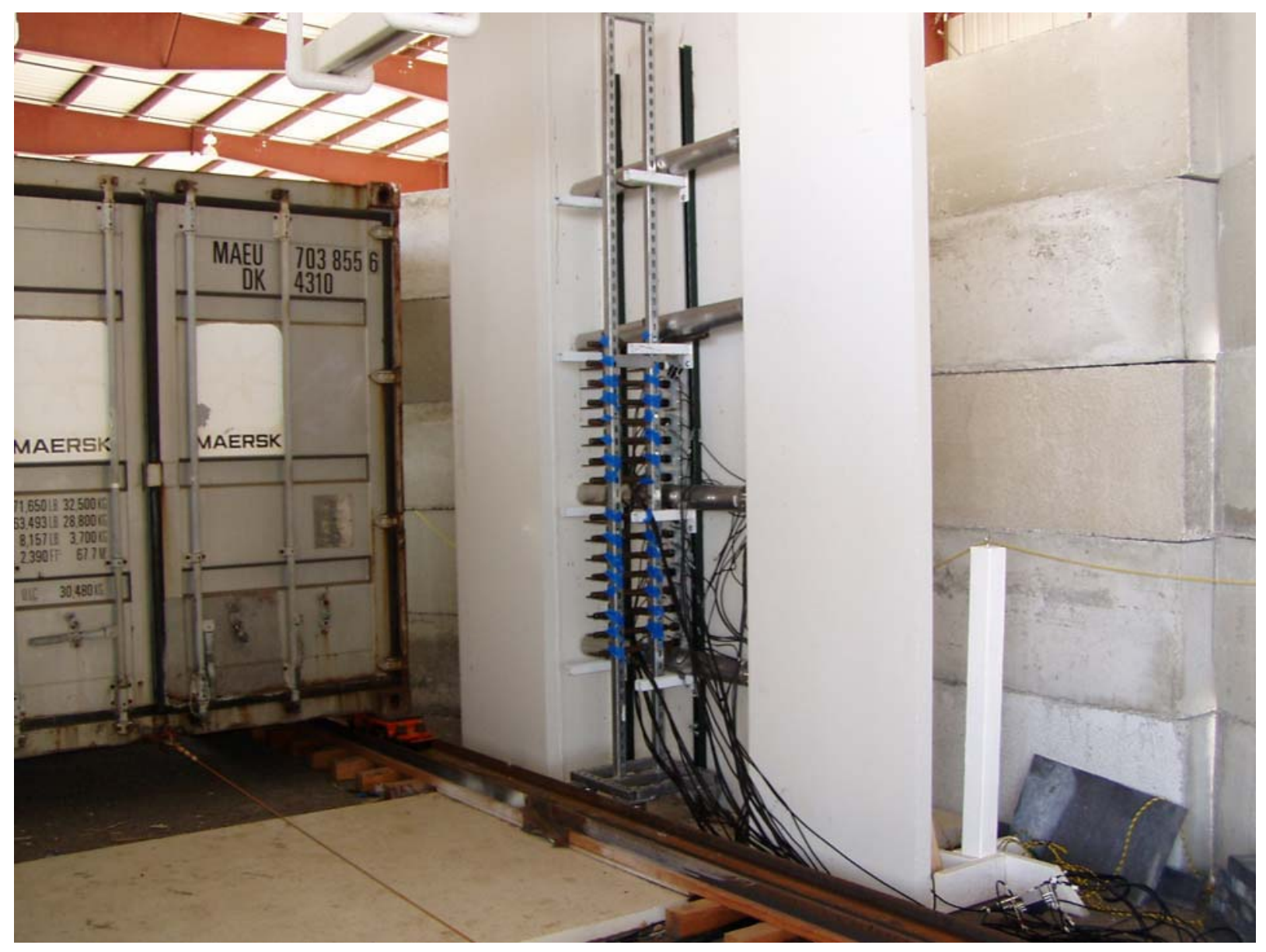

Figure 14. PACECO/ISU/INL gray scale density mapping system mounted with the IAC cargo container translation test assembly.

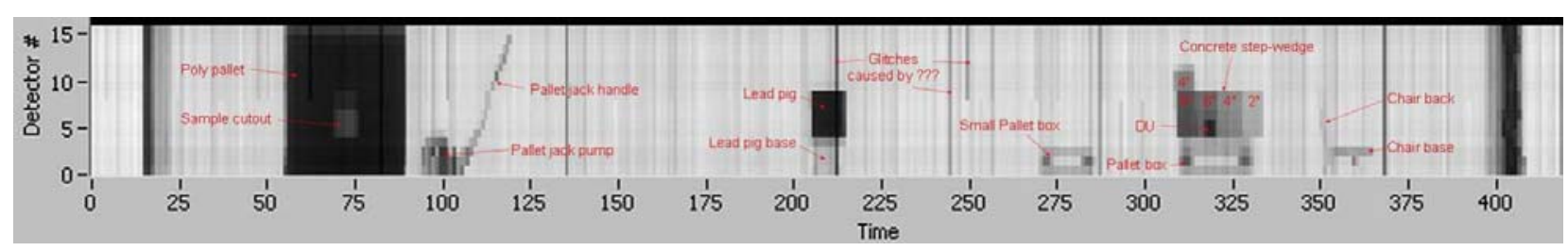

Figure 15. X-ray "image" acquired with the gray scale density mapping system of a 12.2-m (40-ft) cargo container. 


\section{HIGHER ELECTRON BEAM ENERGY INTERROGATIONS}

At the heart of the PPA technology is the electron accelerator. Experimental assessments have been conducted with various electron beam energies showing up to three orders-of-magnitude increases in signature responses from 8 to $24 \mathrm{MeV}$ electron beam operation. ${ }^{12,14}$ The majority of cargo characterizations have been performed with nominal electron beam energies at or below $10 \mathrm{MeV}$ primarily because of current regulations for food inspections. ${ }^{12}$ This energy limit applies to general cargo inspections since food is shipped in inspected cargo; however, the World Health Organization has indicated that higher energy electron beam operations would still be considered. ${ }^{12}$

To obtain higher detection sensitivities with potentially lower cargo dose and address ever higher inspection throughput demands, higher electron beam energy PPA-inspection operations are being investigated. Already technologies utilizing electron beam energies up to $24 \mathrm{MeV}$ are well established and have found considerable industrial and medical applications. Many of these accelerators, especially ones having less than about $8-\mathrm{MeV}$ operations, have even been made field transportable and are used for various radiography applications.

In support of these higher energy enhanced operations, additional energy assessments were performed with the 4.8-kg DU sample and the INL Calibration Pallets. For the DU case, Figure 16 presents the collective results using up to $24-\mathrm{MeV}$ accelerator operations. The test configuration consisted of a 2-m DU-to-photon source and DU-to-neutron detector (PND) distance. The beam axis and the DUto-neutron detector axis were perpendicular, and the DU was aligned at 45-degrees from the beam axis. These experiments were repeated at various times, with various accelerators (Varian's L2500; up to $24 \mathrm{MeV}$ and Varian Clinac: up to $20 \mathrm{MeV}$ ), and at various IAC testing locations (Main IAC Test Cell [TC] and the IAC White Room facility [WR1 and WR2]). While most of the errors involved in these results are systematic, arising from the accelerator operations, it is clear that the largest signal-to-noise ratios are observed with electron beams in the 14 to $20 \mathrm{MeV}$ range. This increased emission trend has been independently observed using thin DU interrogated samples ${ }^{12}$ and with configurations having longer stand-off distances ${ }^{10}$. Note that this energy range corresponds to the photofission interaction peak in nuclear materials. When DU is not present, it is unclear what is driving the increasing detector response above an electron energy of approximately $15 \mathrm{MeV}$. It was initially thought that the $\mathrm{O}^{18}(\gamma, \mathrm{p}) \mathrm{N}^{17}$ with its

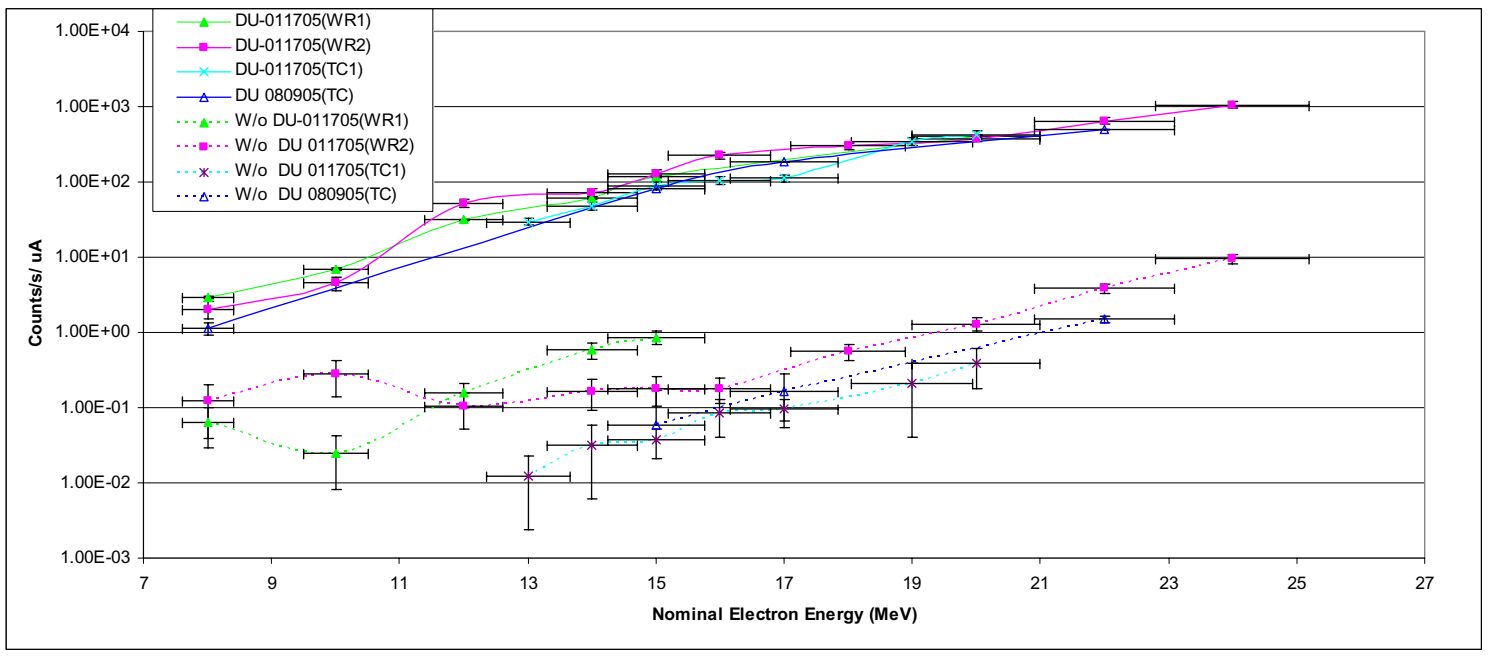

Figure 16. The energy-dependent PND response with and without the 4.8-kg DU plates using various beam currents, accelerators, and testing locations/facilities. (Assumes a $\pm 10 \%$ systematic beam energy error and uses a perpendicular, 2-m DU-to-accelerator and DU-to-detector test configuration.) 
photonuclear threshold energy of about $15.9 \mathrm{MeV}$ may be a contributing factor even though its interaction cross section is relatively small $(\sim 6 \mathrm{mb}$ at $24 \mathrm{MeV})$. This was a good reaction candidate since the resulting $\mathrm{N}^{17}$ decays with a response that is very similar to the characteristic signature used by the PPA system to detect nuclear materials of interest: $\sim 4-6 \mathrm{~s}$ decay times and neutron emissions having a 0.1 - to $1-\mathrm{MeV}$ energy range (for this reaction it is 0.4 to $1.0 \mathrm{MeV}$ ). Initial studies using selective photon source shielding to reduce the effective irradiation volumes have not confirmed this reaction's dominance. Another potential candidate for this background anomaly focuses on the PND design. The PND design was originally optimized for electron beam energies up to $10 \mathrm{MeV}$. A detailed characterization is needed for operation at these higher beam energies. In addition, other possible reaction contributions or system effects are under investigation.

Unfortunately, the data collected during the higher energy Calibration Pallet assessment testing, including the significantly shielded (lead and borated polyethylene) "Challenge" Pallet, resulted in inconclusive data. These inconclusive results are attributed, at least, in part to the lower than expected accelerator beam currents and some uncertainties regarding the electron beam energies. Planning is underway to re-evaluate the INL Calibration Pallet characterizations. However, this set of higher energy Calibration Pallet tests was beneficial since it identified that the use of ionization tubes (such as GM tubes) have considerable difficulties in fully counting and discriminating nuclear material gamma-ray signals during higher energy $(>10 \mathrm{MeV})$ interrogations. The higher energy interrogations produce high photoneutron yields that, after a sufficient slowing down time ( several milliseconds), lead to significant neutron capture gamma rays. These capture events compete with the delayed gamma-ray emissions from a nuclear material detection within a GM tube. Gamma-ray detection system optimization efforts are needed for these higher energy applications.

As previously mentioned, additional assessments with the Calibration Pallets are needed to better understand the various shielding effects at these higher beam energies and to fully maximize the PPA technology's overall detection sensitivity for concealed nuclear material. However, Figure 16 clearly shows the enveloping performance range expected whenever shield material is introduced. For example, using a comparable inspection configuration ${ }^{14}$ and comparable data units, Figure 17 shows this significant increase in detection sensitivity for the 4.8-kg DU nuclear material and the INL Wood Calibration pallet. (Remember, the detection with the nominal $10-\mathrm{MeV}$ operation is already well established).

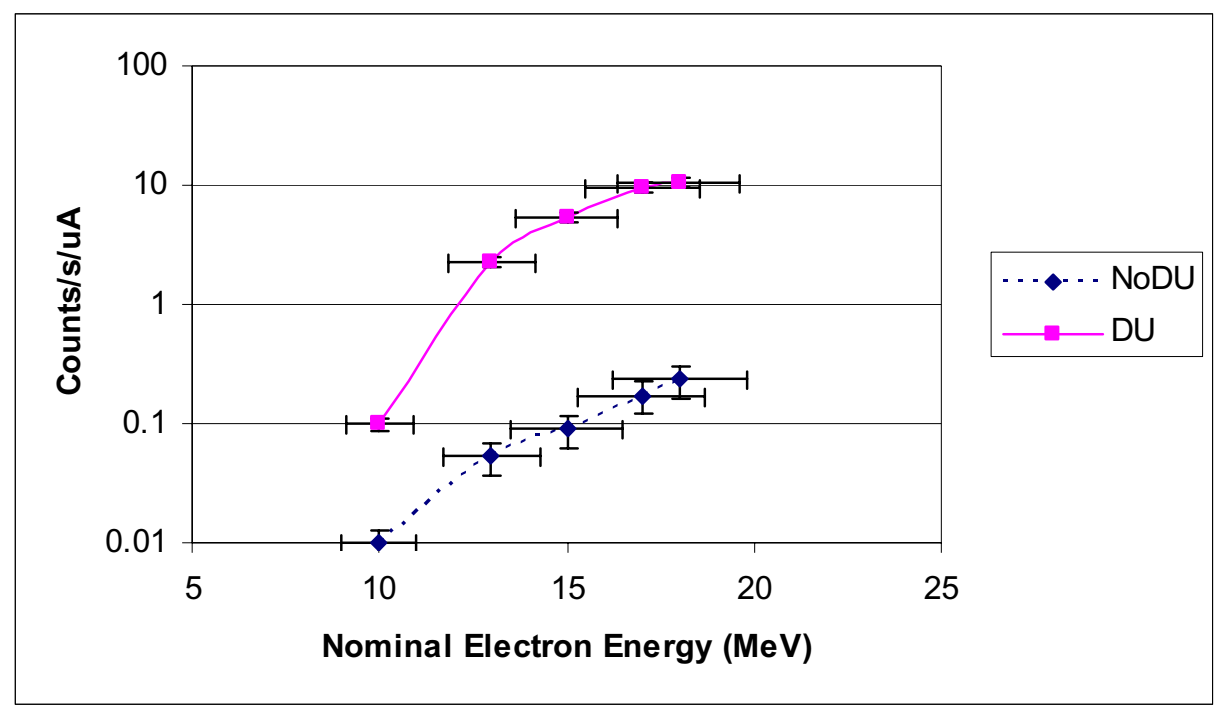

Figure 17. The energy-dependent PND response for the INL Wood Calibration Pallet with and without the $4.8-\mathrm{kg}$ DU plates. 


\section{SUMMARY AND RECOMMENDATIONS}

A prototype nuclear material detection system has been developed by INL, LANL, and the ISU/IAC, employing the PPA technology concept for the inspection of large transportation vehicles such as trucks, cargo containers and railcar containers. This technology development continues to demonstrate its superior capability in addressing the formidable problem of detecting the presence of shielded nuclear materials, especially HEU, and its flexibility in integrating with different detection technologies. The developed system uses energetic 8 to $12 \mathrm{MeV}$ photons (i.e., a nominal $10-\mathrm{MeV}$ electron accelerator) but the $14-18 \mathrm{MeV}$ range still appears to provide optimal detection sensitivity. This energy range provides good cargo material penetration and subsequent photonuclear interactions to stimulate nuclear fissions in concealed nuclear materials. The PPA concept integrates several detection technologies that perform delayed neutron and gamma-ray detection between accelerator pulses to enable nuclear material detection and incorporates a GSDM system to help identify very dense or significant quantities of shield materials that could conceal nuclear material.

The major radiation detection components of the prototype, 10-MeV PPA inspection system were built in CY04. During CY05, these components were integrated using a custom-developed, National Instruments ${ }^{\mathrm{TM}}$-type user interface. These integrated components were assembled and tested with a realistic cargo container testing configuration using a rectangular, 4.8-kg DU sample enclosed within most of the INL Calibration Pallets. Each pallet (with the nuclear material) was aligned with the interrogating beam and positioned in the center of a cargo container inspection configuration so that the nuclear material would have the largest possible material-to-detector distances. Based on $120-\mathrm{s}, 125-\mathrm{Hz}$ nominal $10-\mathrm{MeV}$ inspections, the nuclear material was successfully detected within each pallet configuration studied.

While the delayed neutron detection component (i.e., PNDs) is optimized for this nominal 10-MeV inspection application, the gamma-ray and GSDM components are not. Both components were originally selected based on operational and economical considerations and their ability to provide an integrated detection capability within this type of photonuclear environment. Additional operational component optimizations are needed to help define a final inspection system design.

Based on the current project status, several items are recommended for continued research and evaluation. The list includes:

1. A gamma-ray detection system optimization (advanced GM-tubes, scintillators, etc.)

2. An optimal electron beam energies evaluation with the INL Calibration pallets based on the highest possible detection sensitivity and minimal forward dose

3. A nuclear material detection characterization based on off-beam-centerline axis placement of the nuclear material (will define beam scanning [rastering] requirements, inspection standoff distance, and beam current requirements)

4. Minimum detection limits (MDL) determination for the prototype system using various uranium quantities, thicknesses, and orientations (Relate data to HEU detection sensitivity)

5. A characterization of the prototype system for nuclear material identification using multiple electron beam energies (e.g., differentiate between HEU and DU)

6. A photonuclear characterization of the neutron yields and energy spectra from various materials in order to support complimentary photon and photoneutron interrogations. 


\section{REFERENCES}

1. S. H. Vegors, Jr, "Preliminary Investigation of a Criticality Monitoring Technique for a Transuranic Waste Incinerator", INEL Report TREE-1285 October 1978

2. S.G. Melton, R. Estep, and E. Peterson, "Design and Calibration of the CWAM System for the Y12 Plant", International Conference on Nuclear and Hazardous Waste Management, SPECTRUM 2000, September, 2000.

3. C.L. Hollas, D.A. Close, and C.E. Moss, "Analysis of Fissionable Material using Delayed Gamma Rays from Fission," Nuclear Instruments and Methods in Physics Research B, 24/25, 1987, 503505.

4. J. L. Jones, "Pulsed Photoneutron Interrogation: The GNT Demonstration System," INEL Report WINCO-1225, October 1994.

5. J. L. Jones, "Material Identification Technology (MIT) Concept Technical Feasibility Study", INEL Report WINCO-1147, September 1993.

6. J. L. Jones, "Detection of Pulsed, Bremsstrahlung-induced, Prompt Neutron Capture Gamma-rays with a HPGe Detector," SPIE International Conference: Neutrons in Research and Industry Proceedings, 2867, June 1996, 202-205.

7. J. L. Jones, "Proof-of-Concept Assessment of a Photofission-Based Interrogation System for the Detection of Shielded Nuclear Material" INEEL/EXT-2000-01523, November 2000.

8. D. P. Wells, "Cabinet-safe" study of 1-8 MeV Electron Accelerators", Nuclear Instruments and Methods in Physics Research A, 463, 2001, 118-221.

9. J. L. Jones, "Photofission-based, "Nuclear Material Detection: Technology Demonstration", INEEL Report INEEL/EXT-02-01406, December 2002.

10. J. L. Jones, "Remote Inspections of Cargo Containers for Nuclear Materials: An Initial Experimental and Numerical Assessment”, INEEL Report INEEL/EXT-03-00363, March 2003.

11. M. T. Kinlaw, "Time Dependence of Delayed Neutron Emission for Fissionable Isotopes Identification”, Applied Physics Letters, 86, 254104, 2005.

12. D. R. Norman, "Inspection Applications with Higher Electron Beam Energies", Nuclear Instruments and Methods in Physics Research B, 241, 2005, 787-792.

13. D. R. Norman, "Active Nuclear Material Detection and Imaging”, 2005 IEEE Nuclear Science Symposium Conference, Record N20-2, Puerto Rico, November 2005.

14. J. L. Jones, "Pulsed Photonuclear Assessment (PPA) Technique: CY04 Year-end Progress Report", INL Report INEEL/EXT-05-02583, February 2005.

15. J. L. Jones, "Photonuclear-based, Nuclear Material Detection System for Cargo Containers" Nuclear Instruments and Methods in Physics Research B, 241, 2005, 770-776. 
16. J. L. Jones, "Prototypical Integrated Pulsed Photonuclear Assessment System for Nuclear Material Detection”, 2005 DHS Conference on Working Together with Industry: Research and Development Partnerships in Homeland Security, Conference Proceedings, Boston, Massachusetts, April 27-28, 2005.

17. J. L. Jones, "Detection of Shielded Nuclear Material in a Cargo Container", ANS Accelerator Applications '05 Conference Proceedings, Venice, Italy, August 2005, (to be published in Nuclear Instruments and Methods, 2006).

18. J. S. Hendricks, et. al., "MCNPX, Version 2.5e, "LANL Report, LA-UR-04-0569, February 2004. 


\section{Appendix A}

One-Step Versus Multi-Step Transport Calculation for PPA Applications Using the MCNPX Code 


\title{
Appendix A
}

\section{One-Step Versus Multi-Step Transport Calculation for PPA Applications Using the MCNPX Code}

\begin{abstract}
Currently there are two numerical approaches that could be employed to calculate the delayed neutron detector response for the Pulsed Photonuclear Assessment (PPA) Technique. Both approaches use the LANL, Monte Carlo-based, computer code MCNPX (Publicly released Version 2.5, Reference 18). While it is recognized that other developing versions of MCNPX are available for testing with improved photonuclear capabilities, this Appendix uses the publicly released version.
\end{abstract}

The first approach basically uses a single MCNPX model to calculate a single time-dependent, delayed neutron response. The second approach is similar, but breaks the complex transport problem into two more manageable calculations and includes the time-dependence of the delayed neutron emission in a separate step. Both approaches use a single delta function, or electron impulse, source to start the transport calculations. The second multi-step approach is currently used at the INL and has been successfully benchmarked for the complex and sophisticated PPA-type applications. Both approaches appear to be viable, however, due to current MCNPX code limitations, such as with photofission-induced delayed neutron and gamma-ray production, the former approach with the single time-dependent delayed neutron response function does not appear to be adequate for PPA-type applications. The details of both predictive approaches are discussed in more detail below along with the associated pros and cons.

\section{Numerical Approach No. 1}

Not withstanding MCNPX's inability to address the multi-pulsed nature of the PPA technology, the code can theoretically perform the complete time-dependent transport problem for a single interrogating pulse. This single pulse transport capability would involve the simultaneous and full transport and production of particles that would include: (1) impulse source electrons, (2) bremsstrahlung photons, (3) photoneutrons, (4) photofission events in any nuclear material, (5) thermalization of neutrons, (6) neutron-induced fission events in any nuclear material, (7) scattered photons, neutrons, and electrons, (8) production of radiative capture photons, and (9) production of delayed neutrons and gamma-rays from photofission and neutron-induced fissions. Although theoretically possible with the MCNPX code, this single calculation approach has several transport bottlenecks that limit the code's ability to address PPAtype inspection applications.

First, sufficient source electrons are needed to generate enough bremsstrahlung photons to induce direct photofissions and indirect, neutron-induced fissions resulting from photoneutrons subsequently generated from the bremsstrahlung within the nuclear material and/or its surrounding environment. The number of fissions per source electron is typically $1.0 \mathrm{E}-6$ to $1.0 \mathrm{E}-7$ since the U-235 and U-238 cross sections for photofission ( 0.35 and 0.16 barns max, respectively) and photonuclear neutron yield (1.2 barns) interactions are relatively small. Then, depending on the type of fission event, only one delayed neutron is produced in 30 to 100 fission events. Finally, one of these delayed neutrons must then be transported from the uranium target through a cargo loading configurations (e.g., Calibration Pallet materials) to a Photonuclear Neutron Detector (PND) and induce a detection response. This latter transport step has an efficiency of 1.0E-4 to 1.0E-9 depending on the pallet material and the detector efficiency. Because this is a time-dependent calculation, a time discretization (or time bins) further degrades the statistical error. A calculation of this sort is possible, but requires adequate computer power 
(parallel processing) to fully resolve the detector response. In other words, the full transport calculation is complex and would require sufficient computing resources to alleviate many bottlenecks. Implementation of sophisticated variance reduction techniques would also be necessary for a calculation of this type.

In addition to the transport complexities, special MCNPX photofission libraries would be required for PPA applications. The photofission libraries would need to include both delayed neutron and gammaray production from both U-238 and U-235 (and other nuclear materials of interest). Currently, the MCNPX does not include the production of delayed neutrons/gamma-rays from photofission, and consequentially, the delayed neutron production rate per source electron (or measured beam current) would be under-predicted. In the case of the INL's PPA approach, where the bulk of the fission events are due to photofission events (this is particularly true in the case of our standard DU test target with mostly U-238 atoms present), the delayed neutrons would be significantly under-predicted. MCNPX will however produce a delayed neutron signal, but only from the small neutron fission component and no signal component from the photofission events.

This calculational approach is also based on just a single impulse electron pulse (or accelerator pulse). In actuality, the PPA accelerator system will generate 15,000 pulses (125 Hz operation) in one 120 -second integration period. MCNPX does not have the capability to generate and sample 15,000 square-wave pulses with a $3-\mu$ s pulse width and an 8 -ms separation. However, if a single accelerator pulse response function can be made to calculate the time-dependent, delayed neutron detection response, then this single response curve can be appropriately partitioned and summed to mimic the full 15,000 accelerator pulse response as measured (see Appendix B for additional discussion).

\section{Numerical Approach No. 2}

The second numerical approach is similar to the first, but breaks the transport problem into two time-independent calculations that are more manageable and make it easier to obtain good statistical convergence in a reasonable amount of time with reasonable computing power. The first transport calculation transports a single impulse function of accelerator source electrons into a converter to produce bremsstrahlung photons which in turn produce photoneutrons, photofissions in the DU target, and neutron-induced fission events from the fast and thermalized photoneutrons. The tally result in the DU target is an estimate of the fissions per source electron.

Separate FORTRAN programs then calculate estimates of the number of delayed neutrons emitted from the DU target based on the number of fissions per source electron, the beam current, and delayed neutron yields from both photofission and neutron-induced fissions for both U-235 and U-238. Also included in these estimates is the time-dependent emission of delayed neutrons from the DU target in order to properly time bin the delayed neutrons. Six delayed neutron groups are used with corresponding half-lives and decay constants. This calculation provides the time-dependence for the delayed neutrons emission, accounts for the delayed neutron contributions from all previous accelerator pulses, and mimics the 120 -second time-dependent integration counts measured.

The second transport calculation alleviates one of the bottlenecks mentioned above. This calculation basically estimates the probability of delayed neutrons emitted from the DU target and scoring a count in the PND. Delayed neutrons, assumed to be emitted uniformly from the DU target, are sampled from a delayed neutron energy spectrum. These neutrons are then transported through the DU target, the cargo loading configuration, the cargo container steel walls, the surrounding air, and the PND. The probability of a delayed neutron making it from the DU target to the detector is very small. If the pallet material is borated-polyethylene, for example, the probability is less than one neutron in a billion. This part of the calculation would be very difficult for the first numerical approach to handle and is the main reason the second numerical approach described here was adopted. 


\section{Summary}

Both numerical approaches are similar in nature; both employ MCNPX to perform the transport of the same particles and use a single impulse starting function for the accelerator pulse. However, due to the lack of production of delayed neutrons and gamma-rays from the photofission events and the complexity of a single step transport calculation, MCNPX cannot be expected to accurately predict the delayed signature responses needed for nuclear material detection with the PPA technology. However, the second numerical approach described above (i.e., the INL approach) can account for both photon- and neutroninduced fission delayed neutrons and has been previously benchmarked against actual PPA-type measured data (Reference 14). 


\section{Appendix B}

Single- Versus Multiple-Pulse Effects for the Pulsed Photonuclear Assessment (PPA) Technique 


\section{APPENDIX B}

\section{Single- versus Multiple-Pulse Effects for the Pulsed Photonuclear Assessment (PPA) Technique}

The following discussion attempts to clarify the difference between single pulsed interrogation technology predictions and actual multi-pulsed interrogation technology results. Specifically, this discussion focuses on the multi-pulsed counting technique used in the Pulsed Photonuclear Assessment (PPA) technology at the INL. As will be shown for the PPA application, multi-pulsed sources should always be able to provide increased detection capabilities over a comparable, single-pulsed operation.

One of the primary goals of the PPA technique is to count emitted delayed neutrons following each accelerator bremsstrahlung flash of radiation that induces fissions in a depleted uranium (DU) target. For this discussion, the multi-channel scalar (MCS) is setup with 512 channels corresponding to an $8.0 \mathrm{~ms}$ time period. Delayed neutrons are counted only in the $2-8 \mathrm{~ms}$ time window (or a $75 \%$ live time) so as to avoid the initial "flash effects" or bremsstrahlung-induced prompt effects $(0-2 \mathrm{~ms})$. Otherwise, the chosen 2-8 ms window is not special.

For the sake of discussion, let us assume the accelerator operates at $125 \mathrm{~Hz}$ with an average beam current of $3.0 \mu \mathrm{A}$ having a nominal $10 \mathrm{-MeV}$ electron beam operation. The electron pulse width is $3 \mu \mathrm{s}$ and occurs every $8 \mathrm{~ms}$ during a 120 -second inspection, 15,000 accelerator pulses will be generated. Furthermore, we will assume the DU target is centered in a plywood pallet $(34$ " $\times 40$ " $\times 42$ ") with the DU positioned about one meter from the photon source. Photofissions and neutron-induced fissions are induced in both isotopes (235 and 238) of the uranium target. Also, we'll assume our neutron detectors are $4 \pi$-detectors with a $100 \%$ counting efficiency (so that all delayed neutrons emitted during the counting window time interval are all counted).

First, it is instructive to consider a single accelerator pulse, specifically Pulse No. 1 and forget about the following 14,999 pulses for now. This one pulse will induce approximately 41,155 fission events in the DU; the bulk due to photofission of the U-238. From the delayed neutron yields corresponding to the four components (U238 and U235 photofission and neutron fission), a total of approximately 1,200.4 delayed neutrons will be emitted over all time following the pulse. If we now setup the MCS to sample the time-dependent delayed neutron signal, the response curve is shown in Figure B-1. The MCS performs 15,000 counts, one every $8 \mathrm{~ms}$, with a 2-8 ms acquisition window and resets at the end of each $8 \mathrm{~ms}$ interval.

Figure B-1 shows that in the first 2-8 ms interval 5.539 delayed neutron counts are registered. The MCS resets and in the second 2-8 ms interval 5.445 delayed neutrons are counted. The delayed neutron counts continue to decrease with increasing interval, or "No. of Pulse Time Intervals" as labeled on Figure B-1. If these counts are totaled over the 15,000 intervals (Figure B-1 only shows up to 1,500 intervals), a total of approximately 900 delayed neutrons will have been accounted for, or approximately $75 \%$ of the expected 1,200.4 delayed neutrons to be emitted from the single accelerator pulse.

If we now repeat this experiment, and setup the MCS in the integration mode, we would obtain a response similar to Figure B-2. Here the single pulse delayed neutron signal is summed up and at the end of the $15,000^{\text {th }}$ time interval approximately 900 (or numerically exactly 896.585 ) delayed neutron counts will have been registered. 
Note the delayed neutron response curve of Figure B-1 is really a characteristic decay curve for the emitted delayed neutrons. For the supporting numerical calculations here, the delayed neutrons are conveniently divided into the standard six delayed neutron groups characterized by their own half-life ranging from $172 \mathrm{~ms}$ to 52.38 seconds for the U-238, and $230 \mathrm{~ms}$ to 55.72 seconds for the U-235. Both U-238 photofission and neutron-induced fission events are assumed to have the same group structure, although they have different delayed neutron yields (similarly for the U-235).

In the actual PPA technology, there is not one, but 15,000 accelerator pulses in the 120 -s inspection - one every $8 \mathrm{~ms}$. Delayed neutrons produced in previous pulses contribute to the subsequent pulses and their corresponding count windows. Contributions from these previous pulses are substantial. Consider, for example (and using Figure B-1 data), the second pulse and corresponding 2-8 ms count window, would register $5.539+5.445$ counts, or 10.984 total counts. The 5.539 counts are due to the second accelerator pulse and the 5.445 counts are due to the first accelerator pulse. The total integrated counts in the MCS however are a whopping $5.539+5.539+5.445$ counts, or 16.523 counts, because we now need to include the additional 5.539 delayed counts registered from the first pulse. The integrated counts grow in an exponential manner. Figure B-3 shows the MCS integrated counts as a function of the accelerator pulse number.

The top curve in Figure B-3 is the MCS total integrated delayed neutron counts that we might expect in an actual run, but of course, under our $4 \pi$ detector and $100 \%$ efficiency. At the end of the 15,000 accelerator pulses, the MCS has counted 12,674,400 delayed neutrons (120 second integration count period). The delayed neutrons added up rapidly in the MCS.

Also, plotted in Figure B-3 is a second curve (lower curve). This curve is hypothetical, but is presented to demonstrate a point. The curve presents the integration or summation of only the delayed neutrons produced by that particular pulse (or just the 5.539 delayed neutrons) and no delayed neutron contributions from previous accelerator pulses. Hence the curve is linear and calculated at each accelerator pulse number to have a value of (accelerator pulse no.) * (5.539 delayed neutrons). At the end of the 15,000 pulses, the hypothetical total delayed neutron counts would only be 83,085 counts (or $15,000 * 5.539$ ). The difference between $12,674,400$ and 83,085 counts is huge and clearly shows the importance of the delayed neutron contributions from the previous pulses. This represents a difference of a factor of 152 !

Another way of looking at this issue is by exploring the differences between the total integrated MCS counts using the multi-pulse method (actual PPA method or top curve in Figure B-3) and simply scaling a time-dependent delayed neutron response signal from a single accelerator pulse (for example Figure B-1). A logical question may be: Is it possible to obtain an identical integrated delayed neutron response for $\mathbf{1 5 , 0 0 0}$ accelerator pulses using the delayed neutron response from a single accelerator pulse?

To answer this question, let's first estimate the total delayed neutron counts using a single accelerator pulse and compare it to the total integrated MCS counts we calculated above for an actual run $(12,674,400$ counts $)$.

First, let us estimate the total counts one might expect if a single pulse response is used. If one simply integrates the single accelerator pulse response from $2 \mathrm{~ms}$ to 120 seconds, we would obtain approximately 1,200 delayed neutron counts. Therefore, if we have 15,000 identical pulses overlaid on top of the single response, so as to increase the signal magnitude by a factor of 15,000 , then the total integrated $2 \mathrm{~ms}$ to 120 second delayed neutron counts would be $(15,000 * 1,200)=18,000,000$ delayed neutron counts. However, since the PPA technique's count duty cycle is $75 \%$ [ $(2-8 \mathrm{~ms}) / 8$ $\mathrm{ms}]^{*} 100=75 \%$, we would need to adjust the total counts to $(18,000,000) *(0.75)=13,500,000$ delayed 
neutron counts. Compared to the $12,674,400$ counts registered by a $2-8 \mathrm{~ms}$ acquisition window following each of the 15,000 individual accelerator pulses, this is only $6.5 \%$ larger in magnitude and represents a reasonable means to estimate the exact multi-pulse numerical calculation if one uses all the delayed neutron emissions generated after $2 \mathrm{~ms}$ from an appropriately-scaled, single pulse response.

To better understand why the single response gives a reasonable estimate, let us look at two extreme cases. The first case will produce exact agreement between the two methods and the second case will show that the extreme case is only a factor of two larger or $100 \%$ overestimate of the exact numerical response. Because of the relatively short effective half-life (2-6 s) of the delayed neutron fission response compared to the longer 120-s integration time, the PPA detection method is more representative of the first extreme case.

The first extreme case will assume that the delayed neutron radiation is emitted at $2 \mathrm{~ms}$ after the accelerator pulse has fired and the delayed neutron radiation then decays rapidly and is completely gone by $8 \mathrm{~ms}$. Because of the rapid decay, there will be no delayed neutron contributions from previous pulses. In this case, the single pulse method will estimate the exact number of total delayed neutron counts.

The second extreme case involves a very long and slow decay of the delayed neutron radiation, such that the decay curve (re: Figure B-1) is flat and constant. Therefore, we can assume that the delayed neutron contribution from the present pulse and contributions from each and every previous pulse are identical. Let us call this contribution $(\gamma)$ where $\gamma$ is the delayed counts in the 2-8 ms window, and we can assume $\gamma<<5.539$ counts, although it is not relevant what the value is exactly. Then, the multi-pulse would register the following total integrated counts: $(15,000+14,999+14,998+\ldots \ldots .3+2+1)^{*} \gamma$ over the 120 seconds. The single pulse, on the other hand, would estimate $(15,000 * 15,000) * \gamma$, or twice the number of counts the multi-pulse method would predict.

Hence, the single pulse method will over-predict total integrated counts somewhere between exact and twice the true value. In our case, where the delayed neutrons have relatively fast decay times, the single pulse method using the total time-dependent neutron emissions (corrected for the number of source particles), will produce a reasonable estimate of the actual total MCS-integrated delayed neutron counts using small acquisition windows.

In either extreme case above, the length of the time integration period (e.g. 120 seconds) is independent of the result. The primary dependency appears to be on the decay rate of the radiation being counted. In the actual delayed neutron case with 15,000-pulse, 120-second integration time, and 2-8 ms count window, the dependency would seem to be on both the delayed neutron decay rate and to a lesser extent on the integration time, because of the exponential decay nature of the delayed neutron radiation.

In summary, relative to the PPA technique, good comparison estimates can be obtained if one compares the "total" delayed neutrons (i.e., $2 \mathrm{~ms}$ to $120 \mathrm{~s}$ ) from a single pulse prediction to the experimental data acquired in the 2- to 8 -ms acquisition window after each $125-\mathrm{Hz}$ accelerator pulse during a 120 -s inspection. Hence, a single pulse of sufficient intensity could conceptually provide enough information for effective nuclear material detection. Yet, it must be noted that whatever intensity is considered "large" enough, its utilization must still be technologically feasible in a single pulse. Furthermore, if this source could be pulsed at any frequency, it would again result in a further enhanced detection capability. 


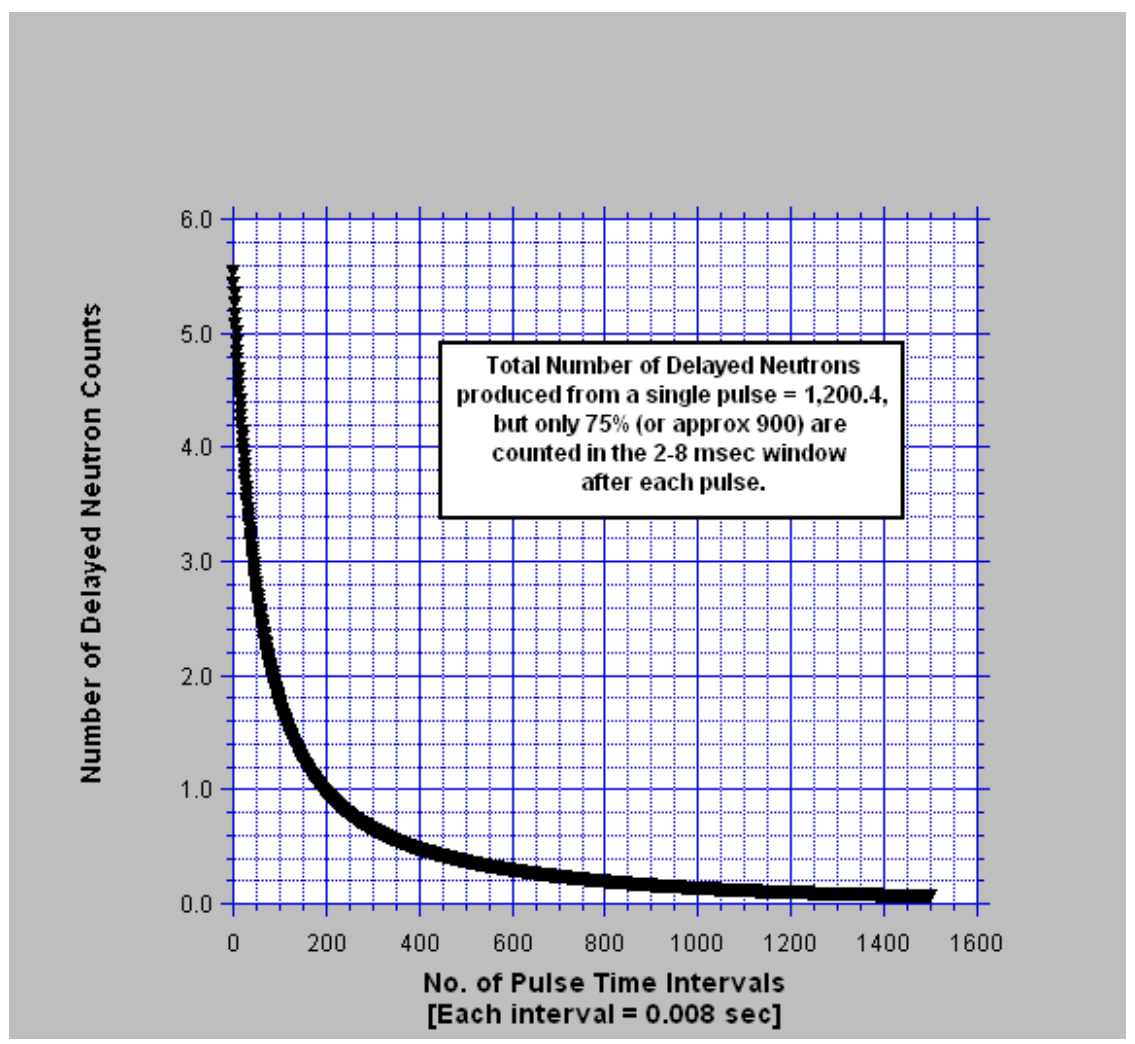

Figure B-1. Number of delayed neutrons counted in subsequent count intervals from a SINGLE accelerator pulse (Pulse No. 1).

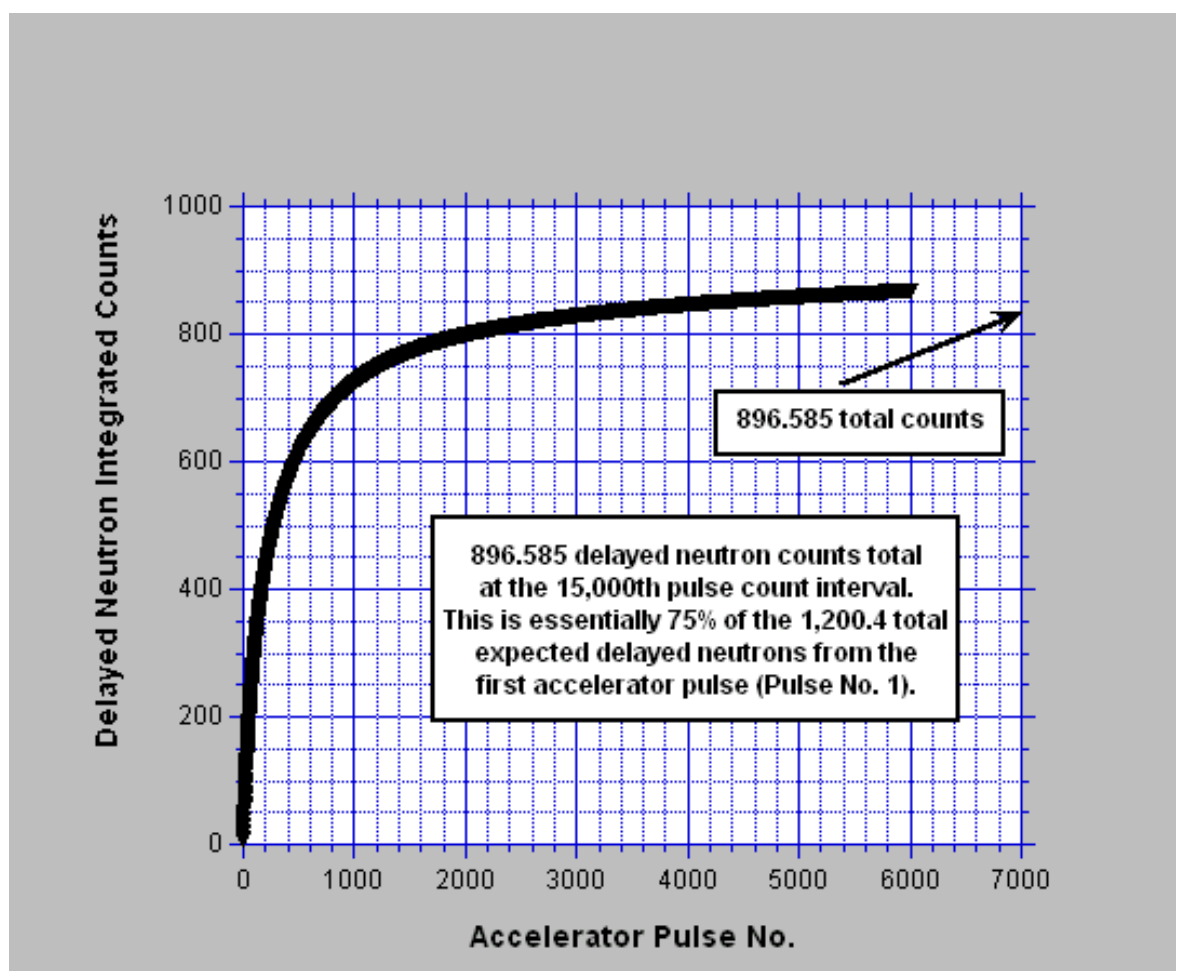

Figure B-2. Integrated delayed neutron counts from a SINGLE accelerator pulse (Pulse No. 1). 


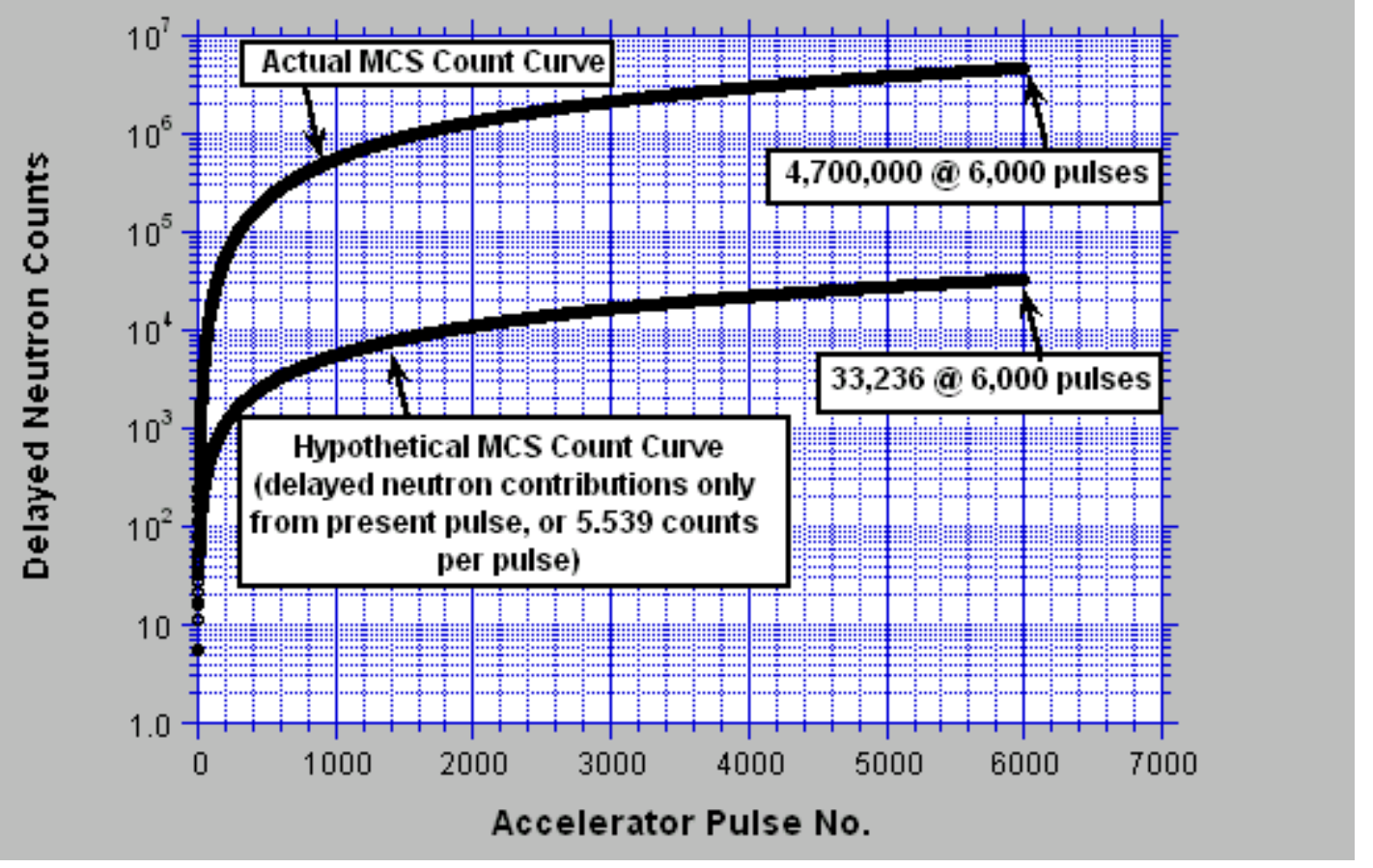

Figure B-3. Actual MCS integration delayed neutron count curve (top curve), plus a hypothetical curve (lower curve) in which delayed neutron contributions from previous pulses are ignored. Note the large difference between the two curves indicative of the important role delayed neutrons from previous pulses play in the total count. 